Pacific Journal of Mathematics

SURGERY AND HANDLE STRAIGHTENING IN HILBER 


\title{
SURGERY AND HANDLE STRAIGHTENING IN HILBERT CUBE MANIFOLDS
}

\author{
T. A. Chapman
}

\begin{abstract}
The main result of this paper establishes an infinite dimensional version of the finite-dimensional handle straightening result of Kirby-Siebenmann. In order to do this a procedure is developed for doing surgery on infinite-dimensional manifolds. These results are used elsewhere to prove that every compact Hilbert cube manifold can be triangulated and to establish the topological invariance of Whitehead torsion.
\end{abstract}

1. Introduction. A Hilbert cube manifold (or $Q$-manifold) is a separable metric space which has an open cover by sets which are homeomorphic to open subsets of the Hilbert cube $Q$. Some obvious examples of $Q$-manifolds are (1) open subsets of $Q$ and (2) $M \times Q$, for any finite-dimensional manifold $M$. Some nonobvious examples of $Q$-manifolds are provided by the work of West [17], where it is shown that $P \times Q$ is a $Q$-manifold, for any locally compact polyhedron $P$. We say that a $Q$-manifold can be triangulated (or is triangulable) provided that it is homeomorphic to $P \times Q$, for some locally compact polyhedron $P$.

The main result of this paper is Theorem 7.1, which gives an infinite-dimensional version of the finite-dimensional handle straightening result of Kirby-Siebenmann [10]. This result is a crucial step in the author's recent proof that Whitehead torsion is a topological invariant [6]. Theorem 7.1 is also used to establish the following theorem. We will use the notation $R^{n}$ for Euclidean $n$-space, and for any $r>0$ we use $B_{r}^{n}=[-r, r]^{n}$ and Int $\left(B_{r}^{n}\right)=(-r, r)^{n}$ to denote the standard $n$-cell of side $2 r$ and its interior.

Theorem. If $X$ is a triangulable $Q$-manifold and $h: R^{n} \times Q \rightarrow X$ is an open embedding, for $n \geqq 2$, then $X \backslash h\left(\operatorname{Int}\left(B_{1}^{n}\right) \times Q\right)$ is triangulable.

The above theorem is the main tool used in proving that every compact Q-manifold can be triangulated. We refer the reader to [5] for details of the proof of this result and for a discussion of its corollaries.

In broad outline the proof of Theorem 7.1 follows the proof of the corresponding finite-dimensional result. In particular, the idea of torus homeomorphisms is used. However to achieve the details we will have to apply a considerable amount of infinite-dimensional machinery. 
A crucial step in the proof of Theorem 7.1 is Theorem 3.1, where we establish an infinite-dimensional surgery result. For the most part, the techniques used in proving Theorem 3.1 differ from those used in corresponding finite-dimensional situations. In particular we don't need Poincaré duality or transversality.

In $\S 2$ we give some definitions and notation which will be used throughout this paper. As mentioned earlier, $\S 3$ is devoted to surgery. In $\S \S 4,5$, and 6 we establish some technical results which will be used in $\$ 7$, where we prove our version of infinite-dimensional handle straightening. Finally in Section 8 we prove the theorem cited earlier.

2. Definitions and notation. In this section we will describe some of the basic terminology that will be used throughout this paper. We will use the representation $Q=\prod_{i=1}^{\infty} I_{i}$, where each $I_{i}$ is the closed interval $[-1,1]$. If $X$ is any space and $k$ is any integer, then we use $p_{k}$ to denote the projection of $X \times Q$ onto $X \times I^{k}$, where $I^{k}=$ $I_{1} \times \cdots I_{k}$. We also let $Q_{k+1}=I_{k+1} \times I_{k+2} \times \cdots$, for all $k>0$. Let $S^{1}$ denote the standard 1-sphere in $R^{2}$ and let $e: R \rightarrow S^{1}$ be the covering projection defined by $e(x)=\exp (\pi i x / 4)$. Let the $n$-torus be denoted by $T^{n}=S^{1} \times \cdots \times S^{1}$ and let $e^{n}: R^{n} \rightarrow T^{n}$ be the product covering projection defined by $e^{n}=e \times \cdots \times e$.

For any space $X$ and $A \subset X$ we use $\operatorname{Int}_{X}(A)$ and $\mathrm{Bd}_{X}(A)$ to denote the topological interior and boundary, respectively, of $A$ in $X$. As usual, the subscript will be omitted when the meaning is clear. If $M$ is an $n$-manifold (i.e. a finite-dimensional manifold), then $\partial M$ will denote the combinatorial boundary of $M$. We will use definitions and notation from [8] concerning $P L$ spaces and manifolds, regular neighborhood theory, etc.

A map is a continuous function and embedding $f: X \rightarrow Y$ is a homeomorphism of $X$ onto $f(X) \subset Y$. When we say that $f: X \rightarrow Y$ is a homeomorphism, we mean that $f$ is a homeomorphism of $X$ onto $Y$. If $f, g: X \rightarrow Y$ are homotopic maps, then we write $f \cong g$. We will denote composition of maps by juxtaposition.

We will also need Anderson's notion of Property $Z$ [1]. A closed subset $A$ of a space $X$ is said to be a $Z$-set in $X$ provided that given any nonnull, homotopically trivial open subset $U$ of $X, U \backslash A$ is also nonnull and homotopically trivial. $Z$-sets in $Q$-manifolds are important because of the following result [2]. Homeomorphism Extension Theorem. Let $K_{1}, K_{2}$ be compact $Z$-sets in a Q-manifold $X$ and let $h$ : $K_{1} \rightarrow K_{2}$ be a homeomorphism such that $h \cong$ id (i.e. $h$ is homotopic to the inclusion of $K_{1}$ into $X$ ). Then $h$ can be extended to a homeomorphism of $X$ onto itself.

3. Infinite-dimensional surgery. Our main result is Theorem 
3.1, where we establish an infinite-dimensional surgery result. In Theorem 3.2 we apply a special case of Theorem 3.1 to prove a result which will be used directly in $\S 7$ for handle straightening.

Let $P$ be a compact connected $P L$ space such that $P$ is 1-connected or $\pi_{1}(P)=Z, M$ be a finite-dimensional $P L$ manifold, and let $\phi: P \times Q \times R \rightarrow M \times Q$ be an open embedding. We will use this notation throughout this section. The proof of Theorem 3.1 will be made more readable by establishing Lemmas $3.1-3.5$, each of which strengthens the preceding one. In the argument that follows we have explicitly given the details for the case $\pi_{1}(P)=Z$. The case in which $P$ is 1-connected is quite similar, and easier.

LEMmA 3.1. There exists an integer $k>0$ and a compact $P L$ submanifold $X$ of $M \times I^{k}$ such that

(1) $X$ is connected,

(2) $\mathrm{Bd}(X)$ is a $P L$ submanifold of $M \times I^{k}$ which is $P L$ bicollared ${ }^{1}$,

(3) $\mathrm{Bd}(X)$ consists of exactly two components,

(4) $\phi(P \times Q \times\{0\}) \subset \operatorname{Int}(X) \times Q_{k+1} \subset X \times Q_{k+1} \subset \dot{\phi}(P \times Q \times R)$.

Proof. Choose an integer $k_{1}>0$ large enough so that

$$
p_{k_{1}} \phi(P \times Q \times\{0\}) \times Q_{k_{1}+1} \subset \phi(P \times Q \times R) .
$$

Choose an open set $U \subset M \times I^{k_{1}}$ such that

$$
p_{k_{1}} \phi(P \times Q \times\{0\}) \times Q_{k_{1}+1} \subset U \times Q_{k_{1}+1} \subset \phi(P \times Q \times R) .
$$

Then let $X_{1} \subset U$ be a regular neighborhood of any compact connected $P L$ subspace of $U$ which contains $p_{k_{1}} \phi(P \times Q \times\{0\})$, and such that $X_{1}$ meets $\partial U$ regularly. Clearly $X_{1}$ is a compact connected $P L$ submanifold of $M \times I^{k_{1}}$ such that $\mathrm{Bd}\left(X_{1}\right)$ is a $P L$ submanifold which is $P L$ bicollared and

$$
\phi(P \times Q \times\{0\}) \subset \operatorname{Int}\left(X_{1}\right) \times Q_{k_{1}+1} \subset X_{1} \times Q_{k_{1}+1} \subset \phi(P \times Q \times R) .
$$

$\mathrm{Bd}\left(X_{1}\right) \times Q_{k_{1}+1}$ is compact and since $P$ is connected it follows that each component of $\varphi(P \times Q \times R) \backslash\left(\operatorname{Int}\left(X_{1}\right) \times Q_{k_{1}+1}\right)$ meets $\operatorname{Bd}\left(X_{1}\right) \times$ $Q_{k_{1}+1}$. Thus $\varphi(P \times Q \times R) \backslash\left(\operatorname{Int}\left(X_{1}\right) \times Q_{k_{1}+1}\right)$ has only finitely many components, and it is clear that exactly two of these are unbounded. Let $\left\{A_{1}, \cdots, A_{p}\right\}$ be the collection of bounded components of $\phi(P \times$ $Q \times R) \backslash\left(\operatorname{Int}\left(X_{1}\right) \times Q_{k_{1}+1}\right)$. Choose $k_{2} \geqq k_{1}$ large enough so that

$$
p_{k_{2}}\left(A_{i}\right) \times Q_{k_{2}+1} \subset \dot{\phi}(P \times Q \times R),
$$

for $1 \leqq i \leqq p$. Let $X_{2}^{\prime}=X_{1} \times I_{k_{1}+1} \times \cdots \times I_{k_{2}}$ and note that $p_{k_{2}}\left(A_{i}\right)$

$1 \mathrm{Bd}(X)$ is $P L$ bicollared means that there exists a $P L$ open embedding $h: \mathrm{Bd}(X) \times$ $(-1,1) \rightarrow M \times I^{k}$ such that $h(x, 0)=x$, for all $x \in \mathrm{Bd}(X)$. 
is a relatively open subset of $\left(M \times I^{k_{2}}\right) \backslash \operatorname{Int}\left(X_{2}^{\prime}\right)$, for $1 \leqq i \leqq p$. Put

$$
X_{2}=X_{2}^{\prime} \cup\left(\bigcup_{i=1}^{p} p_{k_{2}}\left(A_{i}\right)\right)
$$

and note that $X_{2}$ is a compact connected $P L$ submanifold of $M \times I^{k_{2}}$ such that $\mathrm{Bd}\left(X_{2}\right)$ is a $P L$ submanifold of $M \times I^{k_{2}}, \mathrm{Bd}\left(X_{2}\right) P L$ bicollared, $\phi(P \times Q \times\{0\}) \subset \operatorname{Int}\left(X_{2}\right) \times Q_{k_{2}+1} \subset X_{2} \times Q_{k_{2}+1} \subset \phi(P \times Q \times R)$, and $\phi(P \times$ $Q \times R) \backslash\left(\operatorname{Int}\left(X_{2}\right) \times Q_{k_{2}+1}\right)$ has exactly two components.

Let $A, B$ denote the components of $\phi(P \times Q \times R) \backslash\left(\operatorname{Int}\left(X_{2}\right) \times Q_{k_{2}+1}\right)$. We will show how to reduce the number of components of $\left(\operatorname{Bd}\left(X_{2}\right) \times\right.$ $\left.Q_{k_{2}+1}\right) \cap A$. The procedure for reducing the number of components of $\left(\mathrm{Bd}\left(X_{2}\right) \times Q_{k_{2}+1}\right) \cap B$ will be similar. Thus choose distinct components $C, D$ of $\left(\operatorname{Bd}\left(X_{2}\right) \times Q_{k_{2}+1}\right) \cap A$. We can find a path $\sigma$ in $A$ from $C$ to $D$ (i.e. $\sigma \cap C \neq \phi$ and $\sigma \cap D \neq \phi$ ). Choose $k_{3} \geqq k_{2}$ large enough so that $p_{k_{3}}(\sigma) \times Q_{k_{3}+1} \subset A$. Let $X_{3}^{\prime}=X_{2} \times I_{k_{2}+1} \times \cdots \times I_{k_{3}}$ and note that $p_{k_{3}}(\sigma)$ is a path in $\left(M \times I^{k_{3}}\right) \backslash \operatorname{Int}\left(X_{3}^{\prime}\right)$ from $p_{k_{3}}(C)$ to $p_{k_{3}}(D)$. We can use $p_{k_{3}}(\sigma)$ to find a $P L$ arc $\alpha$ in $\left(M \times I^{k_{3}}\right) \backslash\left[\operatorname{Int}\left(X_{3}^{\prime}\right) \cup \partial\left(M \times I^{k_{3}}\right)\right]$ from $p_{k_{3}}(C)$ to $p_{k_{3}}(D)$ such that one endpoint is in $p_{k_{3}}(C)$, the other is in $p_{k_{3}}(D)$, and the remainder of the arc misses $X_{3}^{\prime}$.

In a standard way we can use $\alpha$ to attach a $P L$ handle to $X_{3}^{\prime}$ in $\left(M \times I^{k_{3}}\right) \backslash\left[\operatorname{Int}\left(X_{3}^{\prime}\right) \cup \partial\left(M \times I^{k_{3}}\right)\right]$ which connects the components $p_{k_{3}}(C)$ and $p_{k_{3}}(D)$ (i.e. take a regular neighborhood of $\alpha$ in $\left(M \times I^{k_{3}}\right) \backslash \partial(M \times$ $\left.I^{k_{3}}\right)$ and add it to $X_{3}^{\prime}$ ). In this manner we obtain a compact connected $P L$ submanifold $X_{3}$ of $M \times I^{k_{3}}$ such that $\mathrm{Bd}\left(X_{3}\right)$ is a $P L$ submanifold, $\mathrm{Bd}\left(X_{3}\right)$ is $P L$ bicollared, $\mathrm{Bd}\left(X_{3}\right)$ has one less component than $\mathrm{Bd}\left(X_{3}^{\prime}\right)$, $\phi(P \times Q \times\{0\}) \subset \operatorname{Int}\left(X_{3}\right) \times Q_{k_{3}+1} \subset X_{3} \times Q_{k_{3}+1} \subset \phi(P \times Q \times R)$ and $\phi(P \times$ $Q \times R) \backslash\left(\operatorname{Int}\left(X_{3}\right) \times Q_{k_{3}+1}\right)$ has exactly two components. [For this last condition we need to assume that $k_{3} \geqq 3$.].

It is now clear that we can continue to eliminate boundary components in this manner to inductively arrive at an integer $k \geqq k_{3}$ and a compact connected $P L$ submanifold $X$ of $M \times I^{k}$ which fulfills our requirements.

Our next task is to alter the $X$ obtained in Lemma 3.1 so that, in addition to the properties listed there, if $C$ is a component of $\mathrm{Bd}(X)$, then the inclusion $C \times Q_{k+1} \subset \phi(P \times Q \times R)$ induces an isomorphism of $\pi_{1}\left(C \times Q_{k+1}\right)$ onto $\pi_{1}(\phi(P \times Q \times R))$. In Lemma 3.2 below we achieve the surjectivity of this induced homomorphism and Lemmas 3.3, and 3.4 give an inductive procedure for eliminating the kernel of this homomorphism, while maintaining the surjectivity.

LEMMA 3.2. There exists an inteqer $k>0$ and a compact $P L$ submanifold $X$ of $M \times I^{k}$ such that 
(1) $X$ is connected,

(2) $\mathrm{Bd}(X)$ is a $P L$ submanifold of $M \times I^{k}$ which is $P L$ bicollared,

(3) $\mathrm{Bd}(X)$ consists of exactly two components,

(4) $\phi(P \times Q \times\{0\}) \subset \operatorname{Int}(X) \times Q_{k+1} \subset X \times Q_{k+1} \subset \phi(P \times Q \times R)$,

(5) if $C$ is any component of $\mathrm{Bd}(X)$, then the inclusion $C \times$ $Q_{k+1} \subset \phi(P \times Q \times R)$ induces a surjection of $\pi_{1}\left(C \times Q_{k+1}\right)$ onto $\pi_{1}(\phi(P \times$ $Q \times R))$.

Proof. Using Lemma 3.1 there exists an integer $k_{1}>0$ and a compact connected $P L$ submanifold $X_{1}$ of $M \times I^{k_{1}}$ such that $\mathrm{Bd}\left(X_{1}\right)$ is a $P L$ submanifold of $M \times I^{k_{1}}, \mathrm{Bd}\left(X_{1}\right)$ is $P L$ bicollared and has exactly two components, and $\phi(P \times Q \times\{0\}) \subset \operatorname{Int}\left(X_{1}\right) \times Q_{k_{1}+1} \subset X_{1} \times$ $Q_{k_{1}+1} \subset \phi(P \times Q \times R)$. The procedure is now to "fatten up" $X_{1} \times$ $I_{k_{1}+1} \times \cdots \times I_{k_{2}}$ in $M \times I^{k_{2}}$, for some sufficiently large integer $k_{2}$, so that (5) above is satisfied. Since $\pi_{1}(\phi(P \times Q \times R)=Z$, this amounts to "fattening up" $X_{1} \times I_{k_{1}+1} \times \cdots \times I_{k_{2}}$ so that a loop is introduced into each component of its boundary, where this loop is appropriately chosen to achieve (5) above. We will not give the details of the construction since they are similar to the "fattening up" process used in Lemma 3.5 later on.

LEMMA 3.3. Let $k_{1}>0$ be an integer and let $X_{1} \subset M \times I^{k_{1}}$ be a compact connected $P L$ submanifold of $M \times I^{k_{1}}$ such that $\mathrm{Bd}\left(X_{1}\right)$ is a $P L$ submanifold, $\mathrm{Bd}\left(X_{1}\right)$ is $P L$ bicollared and has exactly two components, and $\phi(P \times Q \times\{0\}) \subset \operatorname{Int}\left(X_{1}\right) \times Q_{k_{1}+1} \subset X_{1} \times Q_{k_{1}+1} \subset \phi(P \times Q \times$ $R)$. Let $C_{1}, D_{1}$ be the components of $\mathrm{Bd}\left(X_{1}\right)$ and let $i_{1}, j_{1}$ be the inclusions $C_{1} \times Q_{k_{1}+1} \subset \phi(P \times Q \times R), D_{1} \times Q_{k_{1}+1} \subset \phi(P \times Q \times R)$. If $\alpha \in \pi_{1}\left(C_{1} \times Q_{k_{1}+1}\right)$ and $\beta \in \pi_{1}\left(D_{1} \times Q_{k_{1}+1}\right)$ satisfy $\left(i_{1}\right)_{*}(\alpha)=1$ and $\left(j_{1}\right)_{*}(\beta)=$ 1 , where $\left(i_{1}\right)_{*}$ and $\left(j_{1}\right)_{*}$ are the induced homomorphisms on the fundamental groups, then there exists an integer $k_{2} \geqq k_{1}$ and a compact $P L$ submanifold $X_{2}$ of $M \times I^{k_{2}}$ such that

(1) $\mathrm{Bd}\left(X_{2}\right)$ is a $P L$ submanifold of $M \times I^{k_{2}}$,

(2) $\mathrm{Bd}\left(X_{2}\right)$ is $P L$ bicollared and has exactly two components,

(3) $\phi(P \times Q \times\{0\}) \subset \operatorname{Int}\left(X_{2}\right) \times Q_{k_{2}+1} \subset X_{2} \times Q_{k_{2}+1} \subset \phi(P \times Q \times R)$,

(4) if $C_{2}, D_{2}$ are the components of $\mathrm{Bd}\left(X_{2}\right)$ (with notation appropriately chosen) and $i_{2}, j_{2}$ are the inclusions $C_{2} \times Q_{k_{2}+1} \subset \phi(P \times Q \times R)$, $D_{2} \times Q_{k_{2}+1} \subset \phi(P \times Q \times R)$, then there exist surjective homomorphisms $u: \pi_{1}\left(C_{1} \times Q_{k_{1}+1}\right) \rightarrow \pi_{1}\left(C_{2} \times Q_{k_{2}+1}\right)$ and $v: \pi_{1}\left(D_{1} \times Q_{k_{1}+1}\right) \rightarrow \pi_{1}\left(D_{2} \times Q_{k_{2}+1}\right)$ such that $u(\alpha)=1, v(\beta)=1,\left(i_{2}\right)_{*} u=\left(i_{1}\right)_{*}$, and $\left(j_{2}\right)_{*} v=\left(j_{1}\right)_{*}$.

Proof. Let $A_{1}, B_{1}$ be the components of $\phi(P \times Q \times R) \backslash\left(\operatorname{Int}\left(X_{1}\right) \times\right.$ $\left.Q_{k_{1}+1}\right)$ and choose notation so that $C_{1} \times Q_{k_{1}+1} \subset A_{1} \subset \phi(P \times Q \times(0, \infty))$ and $D_{1} \times Q_{k_{1}+1} \subset B_{1} \subset \phi(P \times Q \times(-\infty, 0))$. Since $\left(i_{1}\right)_{*}(\alpha)=1$ and $\left(j_{1}\right)_{*}(\beta)=$ 1 we can find maps $f_{1}: B_{1}^{2} \rightarrow \phi(P \times Q \times(0, \infty))$ and $g_{1}: B_{1}^{2} \rightarrow \phi(P \times Q \times$ 
$(-\infty, 0))$ such that $f_{1}\left(\mathrm{Bd}\left(B_{1}^{2}\right)\right) \subset C_{1} \times Q_{k_{1}+1},\left[f_{1} \mid \operatorname{Bd}\left(B_{1}^{2}\right)\right]=\alpha, g_{1}\left(\operatorname{Bd}\left(B_{1}^{2}\right)\right) \subset$ $D_{1} \times Q_{k_{1}+1}$, and $\left[g_{1} \mid \mathrm{Bd}\left(B_{1}^{2}\right)\right]=\beta$ (here [ ] denotes homotopy class). Choose $k_{2} \geqq \max \left(k_{1}, 5\right)$ large enough so that

$$
p_{k_{2}} f_{1}\left(B_{1}^{2}\right) \times Q_{k_{2}+1} \subset \phi(P \times Q \times(0, \infty))
$$

and

$$
p_{k_{2}} g_{1}\left(B_{1}^{2}\right) \times Q_{k_{2}+1} \subset \phi(P \times Q \times(-\infty, 0)) .
$$

Since $k_{2} \geqq 5$ we can approximate $f_{1}$ and $g_{1}$ by $P L$ embeddings $f_{2}: B_{1}^{2} \rightarrow\left(M \times I^{k_{2}}\right) \backslash \partial\left(M \times I^{k_{2}}\right)$

and

$$
g_{2}: B_{1}^{2} \rightarrow\left(M \times I^{k_{2}}\right) \backslash \partial\left(M \times I^{k_{2}}\right)
$$

such that

(1) $f_{2}\left(\mathrm{Bd}\left(B_{1}^{2}\right)\right) \subset C_{1} \times I_{k_{1}+1} \times \cdots \times I_{k_{2}}$ and $g_{2}\left(\mathrm{Bd}\left(B_{1}^{2}\right)\right) \subset D_{1} \times I_{k_{1}+1} \times$ $\cdots \times I_{k_{2}}$,

(2) $f_{2}\left(B_{1}^{2}\right) \times Q_{k_{2}+1} \subset \phi(P \times Q \times(0, \infty))$ and $g_{2}\left(B_{1}^{2}\right) \times Q_{k_{2}+1} \subset \phi(P \times$ $Q \times(-\infty, 0))$,

(3) $\left[\left(\left(f_{2} \mid \mathrm{Bd}\left(B_{1}^{2}\right)\right), 0\right)\right]=\alpha$ and $\left[\left(\left(g_{2} \mid \mathrm{Bd}\left(B_{1}^{2}\right)\right), 0\right)\right]=\beta$, where $\left(\left(f_{2} \mid \mathrm{Bd}\left(B_{1}^{2}\right)\right), 0\right): \mathrm{Bd}\left(B_{1}^{2}\right) \rightarrow C_{1} \times Q_{k_{1}+1}$ and $\left(\left(g_{2} \mid \mathrm{Bd}\left(B_{1}^{2}\right)\right), 0\right): \mathrm{Bd}\left(B_{1}^{2}\right) \rightarrow D_{1} \times$ $Q_{k_{1}+1}$ are defined by $\left(\left(f_{2} \mid \operatorname{Bd}\left(B_{1}^{2}\right)\right), 0\right)(x)=\left(f_{2}(x), 0\right)$ and $\left(\left(g_{2} \mid \operatorname{Bd}\left(B_{1}^{2}\right)\right), 0\right)(x)=$ $\left(g_{2}(x), 0\right)$, where $0=(0,0, \cdots) \in Q_{k_{2}+1}$,

(4) $f_{2}\left(B_{1}^{2}\right)$ is in general position with respect to $C_{1} \times I_{k_{1}+1} \times$ $\cdots \times I_{k_{2}}$,

(5) $g_{2}\left(B_{1}^{2}\right)$ is in general position with respect to $D_{1} \times I_{k_{1}+1} \times$ $\cdots \times I_{k_{2}}$. Choose an open set $U \subset M \times I^{k_{2}}$ such that

$$
\left(C_{1} \times I_{k_{1}+1} \times \cdots \times I_{k_{2}}\right) \cup f_{2}\left(B_{1}^{2}\right) \subset U
$$

and $U \times Q_{k_{2}+1} \subset \phi(P \times Q \times(0, \infty))$. Similarly we can choose an open set $V \subset M \times I^{k_{2}}$ such that

$$
\left(D_{1} \times I_{k_{1}+1} \times \cdots \times I_{k_{2}}\right) \cup g_{2}\left(B_{1}^{2}\right) \subset V
$$

and $V \times Q_{k_{2}+1} \subset \phi(P \times Q \times(-\infty, 0))$. The procedure of Browder now applies here. That is, Lemma 3.1 of [3] implies that we can exchange discs to construct a compact connected $P L$ submanifold $X_{2}$ of $M \times$ $I^{k_{2}}$ such that

(1) $\mathrm{Bd}\left(X_{2}\right)$ is a $P L$ submanifold of $M \times I^{k_{2}}$ which has exactly two components, one in $U$ and the other in $V$,

(2) $\mathrm{Bd}\left(X_{2}\right)$ is $P L$ bicollared,

(3) $\phi(P \times Q \times\{0\}) \subset \operatorname{Int}\left(X_{2}\right) \times Q_{k_{2}+1} \subset X_{2} \times Q_{k_{2}+1} \subset \phi(P \times Q \times R)$,

(4) if $C_{2}$ and $D_{2}$ are the components of $\mathrm{Bd}\left(X_{2}\right)$, where $C_{2} \subset U$ and $D_{2} \subset V$, then the homomorphisms $u$ and $v$ of the statement of the 
lemma exist and satisfy the required properties.

We now use Lemma 3.3 to inductively construct an $X$ which satisfies the requirements of Lemma 3.1 and which also satisfies the property that if $C$ is a component of $\mathrm{Bd}(X)$, then the inclusion $C \times Q_{k+1} \subset \phi(P \times Q \times R)$ induces an isomorphism of $\pi_{1}\left(C \times Q_{k+1}\right)$ onto $\pi_{1}(\phi(P \times Q \times R))$.

LEMmA 3.4. There exists an integer $k>0$ and a compact $P L$ submanifold $X$ of $M \times I^{k}$ such that

(1) $X$ is connected,

(2) $\mathrm{Bd}(X)$ is a PL submanifold of $M \times I^{k}$ which is $P L$ bicollared,

(3) $\mathrm{Bd}(X)$ consists of exactly two components,

(4) $\phi(P \times Q \times\{0\}) \subset \operatorname{Int}(X) \times Q_{k+1} \subset X \times Q_{k+1} \subset \phi(P \times Q \times R)$,

(5) if $C$ is a component of $\mathrm{Bd}(X)$, then the inclusion $C \times Q_{k+1} \subset$ $\phi(P \times Q \times R)$ induces an isomorphism of $\pi_{1}\left(C \times Q_{k+1}\right)$ onto $\pi_{1}(\phi(P \times$ $Q \times R))$.

Proof. Using Lemma 3.2 there exists an integer $k_{1}>0$ and a compact connected $P L$ submanifold $X_{1}$ of $M \times I^{k_{1}}$ such that $\mathrm{Bd}\left(X_{1}\right)$ is a $P L$ submanifold of $M \times I^{k_{1}}, \mathrm{Bd}\left(X_{1}\right)$ is $P L$ bicollared and has exactly two components, and $\phi(P \times Q \times(0\}) \subset \operatorname{Int}\left(X_{1}\right) \times Q_{k_{1}+1} \subset X_{1} \times$ $Q_{k_{1}+1} \subset \phi(P \times Q \times R)$. Moreover if $C_{1}$ and $D_{1}$ are the components of $\mathrm{Bd}\left(X_{1}\right)$ and $i_{1}: C_{1} \times Q_{k_{1}+1} \subset \phi(P \times Q \times R), j_{1}: D_{1} \times Q_{k_{1}+1} \subset \phi(P \times Q \times R)$ are inclusions, then $\left(i_{1}\right)_{*}: \pi_{1}\left(C_{1} \times Q_{k_{1}}\right) \rightarrow \pi_{1}(\phi(P \times Q \times R))$ and $\left(j_{1}\right)_{*}$ : $\pi_{1}\left(D_{1} \times Q_{k_{1}+1}\right) \rightarrow \pi_{1}(\phi(P \times Q \times R))$ are surjections. Let $\alpha$ be a generator of $\pi_{1}(\phi(P \times Q \times R))$ and choose $\alpha_{1} \in \pi_{1}\left(C_{1} \times Q_{k_{1}+1}\right), \beta_{1} \in \pi_{1}\left(D_{1} \times Q_{k_{1}+1}\right)$ such that $\left(i_{1}\right)_{*}\left(\alpha_{1}\right)=\alpha$ and $\left(j_{1}\right)_{*}\left(\beta_{1}\right)=\alpha$. Since $\pi_{1}\left(C_{1} \times Q_{k_{1}+1}\right)$ and $\pi_{1}\left(D_{1} \times Q_{k_{1}+1}\right)$ are finitely generated we can choose generators $\gamma_{1}, \cdots$, $\gamma_{m}$ of $\pi_{1}\left(C_{1} \times Q_{k_{1}+1}\right)$ and $\delta_{1}, \cdots, \delta_{n}$ of $\pi_{1}\left(C_{1} \times Q_{k_{1}+1}\right)$. We now apply Lemma 3.3 to inductively reduce the number of generators of $\pi_{1}\left(C_{1} \times\right.$ $\left.Q_{k_{1}+1}\right)$ and $\pi_{1}\left(D_{1} \times Q_{k_{1}+1}\right)$.

Note that $\left(i_{1}\right)_{*}\left(\gamma_{1}\right)=\alpha^{r}$ and $\left(j_{1}\right)_{*}\left(\delta_{1}\right)=\alpha^{s}$, for some integers $r$ and s. Thus $\left(i_{1}\right)_{*}\left(\gamma_{1} \alpha_{1}^{-r}\right)=1$ and $\left(j_{1}\right)_{*}\left(\delta_{1} \beta_{1}^{-s}\right)=1$. Using Lemma 3.3 we can choose an integer $k_{2} \geqq k_{1}$ and a compact connected $P L$ submanifold $X_{2}$ of $M \times I^{k_{2}}$ such that $\mathrm{Bd}\left(X_{2}\right)$ is a $P L$ submanifold of $M \times I^{k_{2}}$ which is $P L$ bicollared and has exactly two components, and $\phi\{P \times Q \times$ $\{0\}) \subset \operatorname{Int}\left(X_{2}\right) \times Q_{k_{2}+1} \subset X_{2} \times Q_{k_{2}+1} \subset \phi(P \times Q \times R)$. Moreover if $C_{2}, D_{2}$ are the components (appropriately named) of $\mathrm{Bd}\left(X_{2}\right)$ and $i_{2}: C_{2} \times Q_{k_{2}+1} \subset$ $\phi(P \times Q \times R), j_{2}: D_{2} \times Q_{k_{2}+1} \subset \phi(P \times Q \times R)$ are inclusions, then there exist surjective homomorphisms $u: \pi_{1}\left(C_{1} \times Q_{k_{1}+1}\right) \rightarrow \pi_{1}\left(C_{2} \times Q_{k_{2}+1}\right), v: \pi_{1}\left(D_{1} \times\right.$ $\left.Q_{k_{1}+1}\right) \rightarrow \pi_{1}\left(D_{2} \times Q_{k_{2}+1}\right)$ such that $u\left(\gamma_{1} \alpha_{1}^{-r}\right)=1, v\left(\delta_{1} \beta_{1}^{-s}\right)=1,\left(i_{2}\right)_{*} u=\left(i_{1}\right)_{*}$, and $\left(j_{2}\right)_{*} v=\left(j_{1}\right)_{*}$. We have $\pi_{1}\left(C_{2} \times Q_{k_{2}+1}\right)$ generated by $u\left(\alpha_{1}\right), u\left(\gamma_{1}\right), \cdots, u\left(\gamma_{m}\right)$, where $u\left(\gamma_{1}\right)=\left(u\left(\alpha_{1}\right)\right)^{r}$. Thus $\pi_{1}\left(C_{2} \times Q_{k_{2}+1}\right)$ is generated by $u\left(\alpha_{1}\right), u\left(\gamma_{2}\right), \cdots$, $u\left(\gamma_{m}\right)$. Also we have $\left(i_{2}\right)_{*}\left(u\left(\alpha_{1}\right)\right)=\alpha$. Similarly we have $\pi_{1}\left(D_{2} \times Q_{k_{2}+1}\right)$ generated by $v\left(\beta_{1}\right), v\left(\delta_{2}\right), \cdots, v\left(\delta_{n}\right)$ and $\left(j_{2}\right)_{*}\left(v\left(\beta_{1}\right)\right)=\alpha$. 
Inductively continuing this procedure we will eventually arrive at an integer $k_{3} \geqq k_{2}$ and an appropriate subspace $X_{3}$ of $M \times I^{k_{3}}$, with boundary components $C_{3}$ and $D_{3}$, and inclusions $i_{3}: C_{3} \times Q_{k_{3}+1} \subset \phi(P \times$ $Q \times R)$ and $j_{3}: D_{3} \times Q_{k_{3}+1} \subset \phi(P \times Q \times R)$, such that $\pi_{1}\left(C_{3} \times Q_{k_{3}+1}\right)$ is generated by an element $\alpha_{3}$ which satisfies $\left(i_{3}\right)_{*}\left(\alpha_{3}\right)=\alpha$ and $\pi_{1}\left(D_{3} \times\right.$ $\left.Q_{k_{3}+1}\right)$ is generated by an element $\beta_{3}$ which satisfies $\left(j_{3}\right)_{*}\left(\beta_{3}\right)=\alpha$. This implies that $\left(i_{3}\right)_{*}$ and $\left(j_{3}\right)_{*}$ are isomorphisms.

We now prove that the $X$ of Lemma 3.1 can be constructed so that the inclusion $X \times Q_{k+1} \subset \phi(P \times Q \times R)$ is a homotopy equivalence.

LEMMA 3.5. There exists an integer $k>0$ and a compact connected $P L$ submanifold $X$ of $M \times I^{k}$ such that

(1) $\mathrm{Bd}(X)$ is a PL submanifold of $M \times I^{k}$ which has exactly two components,

(2) $\mathrm{Bd}(X)$ is $P L$ bicollared,

(3) $\phi(P \times Q \times\{0\}) \subset \operatorname{Int}(X) \times Q_{k+1} \subset X \times Q_{k+1} \subset \dot{\phi}(P \times Q \times R)$,

(4) the inclusion $X \times Q_{k+1} \subset \phi(P \times Q \times R)$ is a homotopy equivalence.

Proof. Using Lemma 3.4 we can find an integer $k_{1}>0$ and a compact connected $P L$ submanifold $X_{1}$ of $M \times I^{k_{1}}$ such that $\mathrm{Bd}\left(X_{1}\right)$ is a $P L$ submanifold of $M \times I^{k_{1}}$ which has exactly two components, Bd $\left(X_{1}\right)$ is $P L$ bicollared, $\phi(P \times Q \times\{0\}) \subset \operatorname{Int}\left(X_{1}\right) \times Q_{k_{1}+1} \subset X_{1} \times Q_{k+1} \subset$ $\phi(P \times Q \times R)$, and if $C_{1}, D_{1}$ are the components of $\mathrm{Bd}\left(X_{1}\right)$, then the inclusions $C_{1} \times Q_{k_{1}+1} \subset \dot{\phi}(P \times Q \times R)$ and $D_{1} \times Q_{k_{1}+1} \subset \phi(P \times Q \times R)$ induce isomorphisms on $\pi_{1}$. Let $A_{1}, B_{1}$ denote the components of $\phi(P \times$ $Q \times R) \backslash\left(\operatorname{Int}\left(X_{1}\right) \times Q_{k_{1}+1}\right)$, where notation is chosen so that $\operatorname{Bd}\left(A_{1}\right)=$ $C_{1} \times Q_{k_{1}+1}$ and $\mathrm{Bd}\left(\mathrm{B}_{1}\right)=D_{1} \times Q_{k_{1}+1}$.

Consider the following diagram, where all the homomorphisms are induced by inclusions.

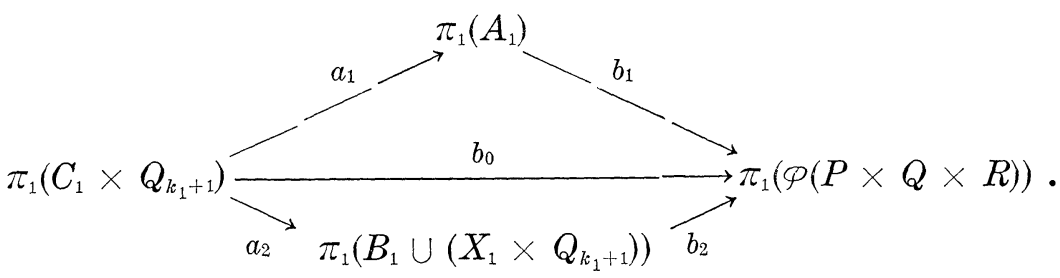

We are given that $b_{0}$ is an isomorphism, thus $a_{1}, a_{2}$ are one-to-one and $b_{1}, b_{2}$ are onto. If $G$ denotes the amalgated free product of $\pi_{1}\left(A_{1}\right)$ and $\pi_{1}\left(B_{1} \cup\left(X_{1} \times Q_{k_{1}+1}\right)\right)$, with the subgroup $a_{1}\left(\pi_{1}\left(C_{1} \times Q_{k_{1}+1}\right)\right)$ of $\pi_{1}\left(A_{1}\right)$ amalgated with subgroup $a_{2}\left(\pi_{1}\left(C_{1} \times Q_{k_{1}+1}\right)\right)$ of $\pi_{1}\left(B_{1} \cup\left(X_{1} \times Q_{k_{1}+1}\right)\right)$, then there exist monomorphisms $c_{0}, c_{1}, c_{2}$ such that the two triangles commute in the following diagram (see [11], p. 32). 


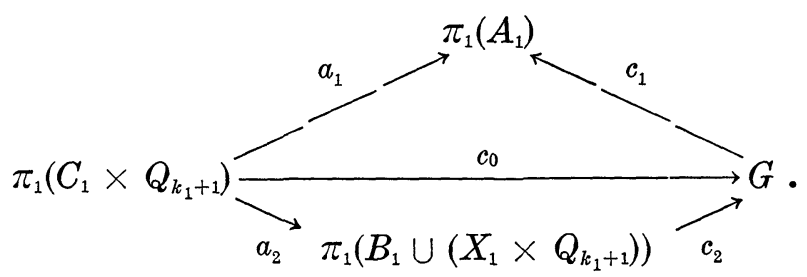

Using the Seifert-Van Kampem theorem there exists a homomorphism $d: \pi_{1}(\varphi(P \times Q \times R)) \rightarrow G$ such that $c_{1}=d b_{1}$ and $c_{2}=d b_{2}$ (see [12], p. 114). Thus $b_{1}, b_{2}$ must be isomorphisms, hence the inclusions $A_{1} \subset \varphi(P \times Q \times$ $R)$ and $B_{1} \subset \varphi(P \times Q \times R)$ induce isomorphisms on $\pi_{1}$.

It follows from [14] (Complement 6.6(b), p. 48) that $A_{1}$ and $B_{1}$ are dominated by finite complexes. It follows from [16] that $A_{1}$ and $B_{1}$ have finite homotopy type if and only if the Wall obstructions $\sigma\left(A_{1}\right)$ and $\sigma\left(B_{1}\right)$ vanish. But since $\pi_{1}\left(A_{1}\right) \cong \pi_{1}\left(B_{1}\right) \cong Z$ it follows that $\sigma\left(A_{1}\right)$ and $\sigma\left(B_{1}\right)$ vanish (see [16], p. 67, for references). Thus $A_{1}$ and $B_{1}$ have finite homotopy type.

Our strategy will be to use the fact that $A_{1}$ and $B_{1}$ have finite homotopy type to "fatten up" $X_{1}$ to get our desired $X$. This is the first time that we really have to exploit the fact that we are dealing with infinite-dimensional spaces. Instead of inductively killing homology groups (as is done in the finite-dimensional situation), we make the jump from $X_{1}$ to $X$ in one step.

Choose a finite complex $K$ and maps $f: A_{1} \rightarrow|K|, g:|K| \rightarrow A_{1}$ which are homotopy inverses. Let $i: C_{1} \rightarrow A_{1}$ be defined by $i(c)=(c, 0)$. Since $C_{1}$ is a compact $P L$ space we can find a $P L$ map $\alpha: C_{1} \rightarrow|K|$ which is homotopic to $f i$ : $C_{1} \rightarrow|K|$. Let $Y$ be the simplicial mapping cylinder of $\alpha$, for appropriate subdivisions of $C_{1}$ and $K$ (see [7] for details). Then $C_{1}$ and $|K|$ have natural identifications in $Y$. Let $r: Y \rightarrow|K|$ be the retraction which collapses the fiber over each point of $|K|$ in $Y$ to that point. With this identification we obviously must have $r \mid C_{1}=\alpha$. Then $r$ is a deformation retraction. Thus $g r: Y \rightarrow A_{1}$ is a homotopy equivalence. Using results from [2] we can find an embedding $h: Y \rightarrow A_{1}$ such that $h(Y)$ is a $Z$-set and $h \cong g r$. Let $j: C_{1} \subset Y$ be inclusion and note that

$$
h j \cong g r j=g \alpha \cong g f i \cong(\mathrm{id}) i=i,
$$

where id denotes the identity. Thus $h \mid C_{1}: C_{1} \rightarrow A_{1}$ is an embedding such that $h \mid C_{1} \cong i$ and $h\left(C_{1}\right)$ is a $Z$-set. Using the Homeomorphism Extension Theorem of $\S 2$ we can adjust $h$ so that the condition $h \mid C_{1}=$ $i$ is additionally satisfied.

Now choose $k_{2} \geqq k_{1}$ large enough so that $p_{k_{2}} h(Y) \times Q_{k_{2}+1} \subset A_{1}$ and let $\beta=p_{k_{2}} h$. If $k_{2}$ is chosen large enough, then we can replace $\beta$ by a $P L$ embedding $\gamma: Y \rightarrow M \times I^{k_{2}}$ such that $\gamma \mid C_{1}=(\mathrm{id}, 0): C_{1} \rightarrow M \times$ 
$I^{k_{2}}$ and $\gamma$ is as close to $\beta$ as we want. Thus $\gamma$ can be chosen so that $\gamma(Y) \times Q_{k_{2}+1} \subset A_{1}$ and $\gamma \cong \beta$. By making one more adjustment to $\gamma$ we can additionally require that

$$
\gamma\left(Y \backslash C_{1}\right) \cap\left(C_{1} \times I_{k_{1}+1} \times \cdots \times I_{k_{2}}\right)=\varnothing .
$$

Note that $(\gamma, 0): Y \rightarrow A_{1}$ is a homotopy equivalence. If $C_{2}=C_{1} \times I_{k_{1}+1} \times$ $\cdots \times I_{k_{2}}$, then there is a deformation retraction of $C_{2} \cup \gamma(Y)$ onto $\left(C_{1} \times\{0\}\right) \cup \gamma(Y)=\gamma(Y)$. Thus the inclusion $\left(C_{2} \cup \gamma(Y)\right) \times Q_{k_{2}+1} \subset A_{1}$ is a homotopy equivalence. Let

$$
N=\left(C_{2} \times I_{k_{2}+1}\right) \cup(\gamma(Y) \times\{1\}) \subset M \times I^{k_{2}+1}
$$

and observe that there is a strong deformation retraction of $N$ onto $\left(C_{2} \cup \gamma(Y)\right) \times\{1\}$, thus the inclusion $N \times Q_{k_{2}+1} \subset A_{1}$ is a homotopy equivalence. Also we have $N \subset \partial\left(\left(M \times I^{k_{2}+1}\right) \backslash \operatorname{Int}\left(X_{3}\right)\right)$, where $X_{3}=$ $X_{1} \times I_{k_{1}+1} \times \cdots \times I_{k_{2}+1}$. Choose a regular neighborhood $N^{*}$ of $N$ in $\partial\left(\left(M \times I^{k_{2}+1}\right) \backslash \operatorname{Int}\left(X_{3}\right)\right)$ such that $N^{*} \times Q_{k_{2}+2} \subset A_{1}$. Clearly the inclusion $N^{*} \times Q_{k_{2}+2} \subset A_{1}$ is a homotopy equivalence. Put $k_{3}=k_{2}+1$.

Since $\left(M \times I^{k_{3}}\right) \backslash \operatorname{Int}\left(X_{3}\right)$ has a boundary collar we can find a $P L$ embedding $\theta: N^{*} \times[0,1] \rightarrow\left(M \times I^{k_{3}}\right) \backslash \operatorname{Int}\left(X_{3}\right)$ such that $\theta(n, 0)=n$, for all $n \in N^{*}$, and $\theta\left(N^{*} \times[0,1]\right) \cap \partial\left(\left(M \times I^{k_{3}}\right) \backslash \operatorname{Int}\left(X_{3}\right)\right)=N^{*}$. By choosing $\theta\left(N^{*} \times[0,1]\right)$ in a neighborhood of $N^{*}$ which is sufficiently close to $N^{*}$, we can additionally assume that $\theta\left(N^{*} \times[0,1]\right) \times Q_{k_{3}+1} \subset$ $A_{1}$. Let $\mathrm{Bd}\left(N^{*}\right)$ denote the boundary of $N^{*}$ in $\partial\left(\left(M \times I^{k_{3}}\right) \backslash \operatorname{Int}\left(X_{3}\right)\right)$ and let $\psi: \operatorname{Bd}\left(N^{*}\right) \times[0,1] \rightarrow N^{*}$ be a boundary collar of $N^{*}$, where $\psi(n, 0)=n$, for all $n \in \mathrm{Bd}\left(N^{*}\right)$. Let us additionally assume that $\psi\left(\operatorname{Bd}\left(N^{*}\right) \times[0,1]\right) \cap N=\varnothing$. Let $\tau: N^{*} \mid \psi\left(\operatorname{Bd}\left(N^{*}\right) \times[0,1 / 2)\right) \rightarrow[0,1]$ be a $P L$ map such that $\tau(N)=\{1\}$ and $\tau^{-1}(0)=\psi\left(\operatorname{Bd}\left(N^{*}\right) \times\{1 / 2\}\right)$. Put $X_{3}^{\prime}=X_{3} \cup G$, where

$$
G=\bigcup\left\{\theta(n, t)\left|n \in N^{*}\right| \psi\left(N^{*} \times[0,1 / 2)\right) \text { and } 0 \leqq t \leqq \tau(n) / 2\right\} .
$$

It is now clear that $X_{3}^{\prime}$ is a compact connected $P L$ submanifold of $M \times I^{k_{3}}$ such that $\mathrm{Bd}\left(X_{3}^{\prime}\right)$ is a $P L$ submanifold which is $P L$ bicollared, $\mathrm{Bd}\left(X_{3}^{\prime}\right)$ has exactly two components, and $X_{1} \times Q_{k_{1}+1} \subset X_{3}^{\prime} \times Q_{k_{3}+1} \subset \phi(P \times$ $Q \times R)$. Let $A_{3}^{\prime}, B_{3}^{\prime}$ be the components of $\phi(P \times Q \times R) \backslash\left(\operatorname{Int}\left(X_{3}^{\prime}\right) \times Q_{k_{3}+1}\right)$ and let $C_{3}^{\prime}, D_{3}^{\prime}$ be the components of $\mathrm{Bd}\left(X_{3}^{\prime}\right)$, where notation is chosen so that $\operatorname{Bd}\left(A_{3}^{\prime}\right)=C_{3}^{\prime} \times Q_{k_{3}+1}$ and $\operatorname{Bd}\left(B_{3}^{\prime}\right)=D_{3}^{\prime} \times Q_{k_{3}+1}$. We can also choose notation so that $B_{3}^{\prime}=B_{1}$ and $D_{3}^{\prime}=D_{1} \times I_{k_{1}+1} \times \cdots \times I_{k_{3}}$. Then it follows that the inclusion $C_{3}^{\prime} \times Q_{k_{3}+1} \subset A_{3}^{\prime}$ is a homotopy equivalence. Thus we have successfully operated on one side of $X_{1}$.

It is clear that the above procedure can be repeated to find an integer $k_{4}>0$ and a compact connected $P L$ submanifold $X_{4}$ of $M \times$ $I^{k_{4}}$ such that $\mathrm{Bd}\left(X_{4}\right)$ is a $P L$ submanifold of $M \times I^{k_{4}}$ which is $P L$ bicollared, $\mathrm{Bd}\left(X_{4}\right)$ has exactly two components, say $C_{4}$ and $D_{4}, \phi(P \times$ 
$Q \times\{0\}) \subset \operatorname{Int}\left(X_{4}\right) \times Q_{k_{4}+1} \subset X_{4} \times Q_{k_{4}+1} \subset \phi(P \times Q \times R)$, and if $A_{4}, B_{4}$ are the components of $\phi(P \times Q \times R) \backslash\left(\operatorname{Int}\left(X_{4}\right) \times Q_{k_{4}+1}\right)$ such that $\operatorname{Bd}\left(A_{4}\right)=$ $C_{4} \times Q_{k_{4}+1}$ and $\mathrm{Bd}\left(B_{4}\right)=D_{4} \times Q_{k_{4}+1}$, then the inclusions $C_{4} \times Q_{k_{k^{+}+1}} \subset$ $A_{4}$ and $D_{4} \times Q_{k_{4}+1} \subset B_{4}$ are homotopy equivalences. A well-known property of ANR's implies that $C_{4} \times Q_{k_{4}+1}$ is a strong deformation retract of $A_{4}$ and $D_{4} \times Q_{k_{4}+1}$ is a strong deformation retract of $B_{4}$ (for example see [15], p. 31). This implies that the inclusion $X_{4} \times$ $Q_{k_{4}+1} \subset \varphi(P \times Q \times R)$ is a homotopy equivalence. Then put $X=X_{4}$ and $k=k_{4}$ to fulfill our requirements.

We are now ready to state and prove our main surgery result. What we are going to do is modify the boundary of the $X$ which was obtained in Lemma 3.5. For the reader who is comparing this process with finite-dimensional surgery, this is the step in which we exchange handles.

THEOREM 3.1. There exists an integer $k>0$ and a compact $P L$ submanifold $X$ of $M \times I^{k}$ such that

(1) $\mathrm{Bd}(X)$ is a $P L$ submanifold of $M \times I^{k}$ which is $P L$ bicollared,

(2) $\mathrm{Bd}(X)$ has exactly two components,

(3) $\phi(P \times Q \times\{0\}) \subset \operatorname{Int}(X) \times Q_{k+1} \subset X \times Q_{k_{1}+1} \subset \dot{\phi}(P \times Q \times R)$,

(4) the inclusion $X \times Q_{k+1} \subset \phi(P \times Q \times R)$ is a homotopy equivalence,

(5) for each component $C$ of $\mathrm{Bd}(X)$, the inclusion $C \times Q_{k+1} \subset$ $\dot{\phi}(P \times Q \times R)$ is a homotopy equivalence.

Proof. Using Lemma 3.5 we can find an integer $k_{1}>0$ and a compact $P L$ submanifold $X_{1}$ of $M \times I^{k_{1}}$ such that

(1) $\mathrm{Bd}\left(X_{1}\right)$ is a $P L$ submanifold of $M \times I^{k_{1}}$ which is $P L$ bicollared,

(2) $\mathrm{Bd}\left(X_{1}\right)$ has exactly two components,

(3) $\phi(P \times Q \times\{0\}) \subset \operatorname{Int}(X) \times Q_{k_{1}+1} \subset X \times Q_{k+1} \subset \phi(P \times Q \times R)$,

(4) the inclusion $X_{1} \times Q_{k_{1}+1} \subset \phi(P \times Q \times R)$ is a homotopy equivalence. Let $A_{1}, B_{1}$ be the components of $\phi(P \times Q \times R) \backslash\left(\operatorname{Int}\left(X_{1}\right) \times\right.$ $\left.Q_{k_{1}+1}\right)$ and let $C_{1}, D_{1}$ be the components of $\mathrm{Bd}\left(X_{1}\right)$ so that $\mathrm{Bd}\left(A_{1}\right)=$ $C_{1} \times Q_{k_{1}+1}$ and $\mathrm{Bd}\left(B_{1}\right)=D_{1} \times Q_{k_{1}+1}$. It is possible that the inclusions $C_{1} \times Q_{k_{1}+1} \subset \dot{\phi}(P \times Q \times R)$ and $D_{1} \times Q_{k_{1}+1} \subset \dot{\phi}(P \times Q \times R)$ are not homotopy equivalences. To remedy this defect we now dig back into $X_{1}$ to produce our required $X$. This amounts to "fattening up" $A_{1}$ and $B_{1}$. We will only give the details for operating on $A_{1}$. A similar procedure will work on $B_{1}$. Choose notation so that we have $A_{1} \subset$ $\phi(P \times Q \times(0, \infty))$ and $B_{1} \subset \phi(P \times Q \times(-\infty, 0))$.

Choose $r>0$ so that $\phi(P \times Q \times[-r, r]) \subset \operatorname{Int}\left(X_{1}\right) \times Q_{k_{1}+1}$. Then the inclusion $\phi(P \times\{0\} \times\{r\}) \subset X_{1} \times Q_{k_{1}+1}$ is a homotopy equivalence, where $0=(0,0, \cdots) \in Q$. Put $P_{0}=\phi(P \times\{0\} \times\{r\})$, let $f: P_{0} \rightarrow X_{1} \times$ $Q_{k_{1}+1}$ be inclusion, and let $g: X_{1} \times Q_{k_{1}+1} \rightarrow P_{0}$ be a map such that $f$ and 
$g$ are homotopy inverses. Let $i: C_{1} \rightarrow X_{1} \times Q_{k_{1}+1}$ be the map defined by $i(c)=(c, 0)$ and let $\alpha: C_{1} \rightarrow P_{0}$ be a PL approximation to $g i$ (i.e., $\alpha \cong g i)$. [We are regarding $P_{0}$ as having a fixed $P L$ structure which is inherited from $P$.] Let $Y$ be the simplicial mapping cylinder of $\alpha$, where $C_{1}$ and $P_{0}$ have natural identifications in $Y$, and let $r: Y \rightarrow P_{0}$ be the map which collapses the fiber over each point of $P_{0}$ to that point. Then $f r: Y \rightarrow X_{1} \times Q_{k_{1}+1}$ is a homotopy equivalence and $f r \mid C_{1}: C_{1} \rightarrow$ $X_{1} \times Q_{k_{1}+1}$ is homotopic to $i: C_{1} \rightarrow X_{1} \times Q_{k_{1}+1}\left(\right.$ in $\left.X_{1} \times Q_{k_{1}+1}\right)$. But $\phi(P \times$ $Q \times[r, \infty))$ is a retract of $\phi(P \times Q \times R)$. Thus the homotopy joining $i$ to $f r \mid C_{1}$ can be realized in $\left(X_{1} \times Q_{k_{1}+1}\right) \cap \phi(P \times Q \times[r, \infty))$. Therefore the main idea of Lemma 3.5 can be used to dig back into $X_{1} \times Q_{k_{1}+1}$ to produce an integer $k_{2} \geqq k_{1}$ and a compact $P L$ submanifold $X_{2}$ of $M \times I^{k_{2}}$ such that

(1) $\phi(P \times Q \times\{0\}) \subset \operatorname{Int}\left(X_{2}\right) \times Q_{k_{2}+1} \subset X_{2} \times Q_{k_{2}+1} \subset X_{1} \times Q_{k_{1}+1}$,

(2) $\mathrm{Bd}\left(X_{2}\right)$ is a $P L$ submanifold of $M \times I^{k_{2}}$ which is $P L$ bicollared,

(3) $\mathrm{Bd}\left(X_{2}\right)$ has exactly two components,

(4) one component of $\mathrm{Bd}\left(X_{2}\right)$ is $D_{1} \times I_{k_{1}+1} \times \cdots \times I_{k_{2}}$,

(5) if $C_{2}$ is the other component of $\mathrm{Bd}\left(X_{2}\right)$, then $C_{2} \times Q_{k_{2}+1} \subset$ $\phi(P \times Q \times R)$ is a homotopy equivalence,

(6) the inclusion $X_{2} \times Q_{k_{2}+1} \subset \phi(P \times Q \times R)$ is a homotopy equivalence. Thus we have successfully operated on $A_{1}$. Using a similar procedure on $B_{1}$ we can produce our desired $X$ and $k$.

In Theorem 3.2 below we establish a special case of Theorem 3.1 which will also be used in the proof of Theorem 7.1.

THEOREM 3.2. Let $M$ be a $P L$ manifold and let $h: R^{n} \times Q \rightarrow M \times$ $Q$ be an open embedding, where $n \geqq 2$. Then there exists an integer $k>0$ and a compact contractible $P L$ submanifold $X$ of $M \times I^{k}$ such that

(1) $\mathrm{Bd}(X)$ is a PL submanifold of $M \times I^{k}$,

(2) $\mathrm{Bd}(X)$ is $P L$ bicollared,

(3) $h\left(B_{1}^{n} \times Q\right) \subset \operatorname{Int}(X) \times Q_{k+1} \subset X \times Q_{k+1} \subset h\left(B_{2}^{n} \times Q\right)$,

(4) the inclusion $\mathrm{Bd}(X) \times Q_{k+1} \subset h\left(\left(B_{2}^{n} \backslash \operatorname{Int}\left(B_{1}^{n}\right)\right) \times Q\right)$ is a homotopy equivalence.

Proof. Let $\phi=h \mid\left(\operatorname{Int}\left(B_{2.5}^{n}\right) \backslash B_{.5}^{n}\right) \times Q$, which may be viewed as an open embedding of $\mathrm{Bd}\left(B_{1}^{n}\right) \times Q \times R$ into $M \times Q$. Using Theorem 3.1 we can find an integer $k>0$ and a compact $P L$ submanifold $Y$ of $M \times I^{k}$ such that $Y$ is $P L$ bicollared, $Y \times Q_{k+1} \subset h\left(\left(\operatorname{Int}\left(B_{2}^{n}\right) \backslash B_{1}^{n}\right) \times Q\right)$, this inclusion is a homotopy equivalence, and $Y \times Q_{k+1}$ separates $h\left(\left(\operatorname{Int}\left(B_{2.5}^{n}\right) \backslash B_{.5}^{n}\right) \times Q\right)$ into two components, one containing $h\left(\operatorname{Bd}\left(B_{1}^{n}\right) \times\right.$ $Q)$ and the other containing $h\left(\mathrm{Bd}\left(B_{2}^{n}\right) \times Q\right)$.

Clearly $h^{-1}\left(Y \times Q_{k+1}\right)$ separates $R^{n} \times Q$ into two components, one component is bounded and contains $B_{1}^{n} \times Q$. Let $C$ be the closure 
of this component and note that $C$ is contractible. Then $X=p_{k} h(C)$ fulfills our requirements.

4. Simple homotopy type. In this section we develop the machinery on simple homotopy type which will be needed in the proof of Theorem 7.1. The first result strengthens a theorem of West [17].

LEMMA 4.1. Let $K$ and $L$ be finite connected complexes and let $f:|K| \rightarrow|L|$ be a simple homotopy equivalence. Then $f \times \mathrm{id}:|K| \times$ $Q \rightarrow|L| \times Q$ is homotopic to a homeomorphism.

Proof. In [17] West proved that $|K| \times Q$ is homeomorphic to $|L| \times Q$. We will modify West's argument to give our desired conclusion. Actually no new ideas are involved and our proof is just West's proof with a little more attention paid to appropriate details.

Using Whitehead [18] there exist pairs $\left(K_{0}, L_{0}\right),\left(K_{1}, L_{1}\right), \cdots,\left(K_{n}\right.$, $L_{n}$ ) of finite complexes such that $K=K_{0}, L_{n}=L$, each $\left|L_{i-1}\right|$ is $P L$ homeomorphic to $\left|K_{i}\right|$, and each $L_{i}$ is a formal deformation of $K_{i}$ (we use the language of [13]). For each $i$ let us define a map $f_{i}:\left|K_{i}\right| \rightarrow$ $\left|L_{i}\right|$ as follows: $f_{i}$ is inclusion if $K_{i} \rightarrow L_{i}$ is a formal expansion and $f_{i}$ is a retraction which is homotopic to the identity if $K_{i} \rightarrow L_{i}$ is a formal contraction. For each $i$ let $g_{i-1}:\left|L_{i-1}\right| \rightarrow\left|K_{i}\right|$ be the given $P L$ homeomorphism. Then $f:|K| \rightarrow|L|$ is homotopic to $f_{n} g_{n-1} f_{n-1} g_{n-2} \cdots$ $f_{1} g_{0} f_{0}$. But in [17] West proved that each $f_{i} \times \mathrm{id}:\left|K_{i}\right| \times Q \rightarrow\left|L_{i}\right| \times$ $Q$ is homotopic to a homeomorphism. This clearly gives us our desired result.

We now use this result to obtain the main result of this section: It is used in the proof of Theorem 7.1.

THEOREM 4.1. Let $K$ and $L$ be finite connected complexes such that the Whitehead group $\mathrm{Wh}\left(\pi_{1}(|K|)\right)=0$, let $X$ be a compact metric space, and let $i: X \rightarrow|K| \times Q, j: X \rightarrow|L| \times Q$ be embeddings such that $i$ and $j$ are homotopy equivalences and $i(X), j(X)$ are $Z$-sets. Then there exists a homeomorphism $f:|K| \times Q \rightarrow|L| \times Q$ such that $f i=j$.

Proof. Let $g:|K| \times Q \rightarrow X$ be a map such that $i$ and $g$ are homotopy inverses and let $h:|K| \rightarrow|K| \times Q$ be defined by $h(x)=(x$, $0)$, where $0=(0,0, \cdots) \in Q$. If $p:|L| \times Q \rightarrow|L|$ is the projection map, then clearly pjgh: $|K| \rightarrow|L|$ is a homotopy equivalence. Since $\mathrm{Wh}\left(\pi_{1}(|K|)\right)=0$ it follows then that $p j g h$ is a simple homotopy equivalence ([18], p. 43). Using Lemma 4.1 it follows that $p j g h \times$ $i d:|K| \times Q \rightarrow|L| \times Q$ is homotopic to a homeomorphism $\phi:|K| \times Q \rightarrow$ $|L| \times Q$. Then $\phi i: X \rightarrow|L| \times Q$ is an embedding such that $\phi i(X)$ is a $Z$-set. It is easy to see that $\phi i \cong j$. Using the Homeomorphism Extension Theorem of $\S 2$ we can therefore adjust $\phi$ to get our desired 
homeomorphism $f:|K| \times Q \rightarrow|L| \times Q$.

5. Immersions. If $X$ and $Y$ are $Q$-manifolds, then we define an immersion to be a map $\alpha: X \rightarrow Y$ which is locally an open embedding. In Lemma 5.1 below we show that if $Y$ is triangulated, then "large" compacta in $X$ lie in triangulated submanifolds of $X$. Of course if $X$ is compact, then this implies that $X$ is triangulated; however our use of this result is for noncompact $X$. Using an argument along the lines of Theorem 8 of [4], one could actually prove that noncompact $X$ are triangulated. We settle for the weaker version, which has a more direct proof.

Lemma 5.1. Let $X$ be a Q-manifold, $M$ be a finite-dimensional $P L$ manifold, and let $\alpha: X \rightarrow M \times Q$ be an immersion. If $K_{0} \subset G \subset$ $K \subset X$, where $K_{0}$ and $K$ are compact, $G$ is open, and $\alpha \mid G$ is one-to-one, then there exists a finite-dimensional $P L$ manifold $N$, a compact $P L$ submanifold $N_{0}$ of $N$, and an embedding $\phi: N \times Q \rightarrow X$ such that

(1) $K_{0} \subset \operatorname{Int}\left(\phi\left(N_{0} \times Q\right)\right) \subset \phi\left(N_{0} \times Q\right) \subset G \subset K \subset \phi(N \times Q)$,

(2) there exists an integer $n>0$ and a $P L$ submanifold $Y$ of $M \times I^{n}$ such that $\alpha \phi\left(N_{0} \times Q\right)=Y \times Q_{n+1}$,

(3) $\alpha \phi \mid N_{0} \times\{0\}: N_{0} \times\{0\} \rightarrow Y \times\{0\}$ is a $P L$ homeomorphism,

(4) for any $\left(n,\left(q_{i}\right)\right) \in N_{0} \times Q$ we have $\alpha \phi\left(n,\left(q_{i}\right)\right)=\left(y,\left(q_{i}\right)\right)$, where $y$ is chosen so that $\alpha \phi(n, 0)=(y, 0)$.

Proof. Note that $\alpha(G)$ is open in $M \times Q$ and $\alpha\left(K_{0}\right)$ is a compact set in $\alpha(G)$. Thus we can find an integer $m>0$ and compact $P L$ submanifolds $M_{0}^{\prime}, M_{1}^{\prime}$ of $M \times I^{m}$ such that $M_{0}^{\prime} \subset \operatorname{Int}\left(M_{1}^{\prime}\right), M_{1}^{\prime} \times Q_{m+1} \subset$ $\alpha(G), \alpha\left(K_{0}\right) \subset M_{0}^{\prime} \times Q_{m+1}$. Since $\alpha$ is an immersion we can choose an open cover $\mathscr{C}$ of $X_{0}=X \backslash(\alpha \mid G)^{-1}\left(M_{0}^{\prime} \times Q_{m+1}\right)$ such that if $U^{*} \in \operatorname{St}(\mathscr{C})$, then $\alpha \mid U^{*}$ is one-to-one, and if $U \in \mathscr{C}$ and $U \cap(\alpha \mid G)^{-1}\left(M_{1}^{\prime} \times Q_{m+1}\right) \neq \varnothing$,

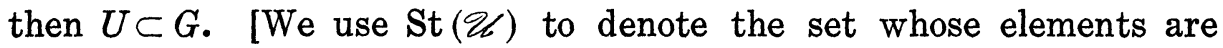
$U\{V \mid V \in \mathscr{U}, V \cap U \neq \varnothing\}$, where $U \in \mathscr{U}$.] We say that an open subset $W$ of $Q$ is a basic open set provided that there exists an integer $k>0$ and an open subset $W^{\prime}$ of $I^{k}$ such that $W=W^{\prime} \times Q_{k+1}$ and such that $W^{\prime}$ is a product of intervals. For each $x \in X_{0}$ we can choose an open subset $A_{x}$ of $X$ such that

$$
x \in A_{x} \subset U_{x} \in \mathscr{C},
$$

for some $U_{x} \in \mathscr{K}$, and such that $\alpha\left(A_{x}\right)$ is the product of an open neighborhood in $M$ with a basic open set in $Q$. Choose a finite subset $\left\{A_{i}\right\}_{i=1}^{p}$ of $\left\{A_{x}\right\}_{x \in X_{0}}$ which covers $K \backslash(\alpha \mid G)^{-1}\left(\operatorname{Int}\left(M_{1}^{\prime}\right) \times Q_{m+1}\right)$. Then we can find an integer $n \geqq m$ large enough so that for each $i$ we have $\alpha\left(A_{i}\right)=B_{i} \times Q_{n+1}$, for some open subset $B_{i}$ of $M \times I^{n}$. Put $M_{1}=$ $M_{1}^{\prime} \times I_{m+1} \times \cdots \times I_{n}$ and let 


$$
\begin{aligned}
N & =\left(\bigcup_{i=1}^{p}\left(\alpha \mid A_{i}\right)^{-1}\left(B_{i} \times\{0\}\right)\right) \cup(\alpha \mid G)^{-1}\left(M_{1} \times\{0\}\right), \\
N_{0} & =(\alpha \mid G)^{-1}\left(M_{1} \times\{0\}\right), \\
Y & =M_{1} .
\end{aligned}
$$

It is clear that the map $p_{n} \alpha \mid N: N \rightarrow M \times I^{n}$, which is locally an open embedding, induces a $P L$ structure on $N$. Thus $N$ is a $P L$ manifold and $N_{0}$ is a $P L$ submanifold of $N$. Let

$$
Z=\left(\bigcup_{i=1}^{p} A_{i}\right) \cup(\alpha \mid G)^{-1}\left(M_{1} \times Q_{n+1}\right)
$$

and define $A_{0}=(\alpha \mid G)^{-1}\left(M_{1} \times Q_{n+1}\right)$. Choose $y \in A_{i}, 0 \leqq i \leqq p$, and define

$$
\psi(y)=\left(\left(\alpha \mid A_{i}\right)^{-1}(b, 0), q\right) \in N \times Q_{n+1},
$$

where $\alpha(y)=(b, q) \in\left(M \times I^{n}\right) \times Q_{n+1}$. It routinely follows that $\psi$ is a homeomorphism of $Z$ onto $N \times Q_{n+1}$. To check this we have to use the properties of the cover $\mathscr{U}$ which are listed at the beginning of this proof. Then $\varphi=\psi^{-1}$ clearly fulfills our requirements (if we identify $Q_{n+1}$ with $Q$ ).

Using the covering projection $e^{n}: R^{n} \rightarrow T^{n}$ of $\S 2$ let $D^{n}=$ $e^{n}\left([2,3]^{n}\right)$ and $E^{n}=e^{n}\left(B_{1}^{n}\right)$. We will find the following result useful in the proof of Theorem 7.1.

LEMmA 5.2. There exists an immersion $\alpha:\left(T^{n} \backslash D^{n}\right) \times Q \rightarrow \operatorname{Int}\left(B_{2}^{n}\right) \times$ $Q$ such that $\alpha\left(e^{n} \times \mathrm{id}\right) \mid B_{1}^{n} \times Q: B_{1}^{n} \times Q \rightarrow B_{1}^{n} \times Q$ is the identity.

Proof. On page 48 of [9] it is shown that there exists a map $\beta: T^{n} \backslash D^{n} \rightarrow \operatorname{Int}\left(B_{2}^{n}\right)$ such that $\beta$ is locally an open embedding and $\beta e^{n} \mid B_{1}^{n}: B_{1}^{n} \rightarrow B_{1}^{n}$ is the identity. Then $\alpha=\beta \times$ id fulfills our requirements.

6. Bounded homeomorphisms. A homeomorphism $h: R^{n} \times Q \rightarrow$ $R^{n} \times Q$ is said to be bounded provided that the set

$$
\left\{\|x-y\| \mid h\left(x, q_{1}\right)=\left(y, q_{2}\right) \text {, for some }\left(x, q_{1}\right) \in R^{n} \times Q\right\}
$$

is bounded above. In this section we describe the apparatus concerning bounded homeomorphisms which will be needed in the proof of Theorem 7.1. Our first result is just an observation due to Kirby.

Lemma 6.1. Let $h: T^{n} \times Q \rightarrow T^{n} \times Q$ be a homeomorphism and let $\tilde{h}: R^{n} \times Q \rightarrow R^{n} \times Q$ be a covering homeomorphism of $h$ which fixes lattice points, i.e. $\tilde{h}\left(\left(a_{i}\right), 0\right)=\left(\left(a_{i}\right), 0\right)$, for all points $\left(a_{i}\right) \in R^{n}$ such that $a_{i} \equiv 4 \bmod 8$. Then $\widetilde{h}$ is a bounded homeomorphism. 
Proof. We just take the argument given on page 44 of [9] and multiply everything by $Q$.

The next simple result gives us conditions which imply the existence of bounded homeomorphisms.

LEMmA 6.2. Using the notation $E^{n}=e^{n}\left(B_{1}^{n}\right)$ (as introduced in $\S 5)$, let $h: T^{n} \times Q \rightarrow T^{n} \times Q$ be a homeomorphism such that $h \mid\left(T^{n} \backslash E^{n}\right) \times$ $\{0\}=\mathrm{id}$, where $n \geqq 2$. Then $h$ can be covered by a bounded homeomorphism $\tilde{h}: R^{n} \times Q \rightarrow R^{n} \times Q$.

Proof. Let $X=\left(T^{n} \backslash E^{n}\right) \times\{0\}$ and note that $\tilde{X}=\left(e^{n} \times \mathrm{id}\right)^{-1}(X)$ is connected, since $n \geqq 2$. Let $\widetilde{h}: R^{n} \times Q \rightarrow R^{n} \times Q$ be the covering homeomorphism of $h$ which satisfies $h((4,4, \cdots, 4), 0)=((4,4, \cdots, 4)$, $0)$. The existence of $\tilde{h}$ follows from standard covering space theory. Since $h \mid X=$ id it follows that $\widetilde{h} \mid \widetilde{X}: \widetilde{X} \rightarrow \widetilde{X}$ is a covering transformation which satisfies $\tilde{h}((4,4, \cdots, 4), 0)=((4,4, \cdots, 4), 0)$. Thus $\tilde{h} \mid \widetilde{X}=$ id, which implies that $\tilde{h}$ fixes lattice points. It now follows from Lemma 6.1 that $\tilde{h}$ is bounded.

We now establish the main result of the section. It is one of the key technical results which will be needed in the proof of Theorem 7.1.

THEOREM 6.1. Let $h: T^{n} \times Q \rightarrow T^{n} \times Q$ be a homeomorphism and let $\widetilde{h}: R^{n} \times Q \rightarrow R^{n} \times Q$ be a covering homeomorphism of $h$ which is bounded. Assume that $A \subset \operatorname{Int}\left(E^{n}\right) \times Q$ is compact, connected, and $h(A) \subset \operatorname{Int}\left(E^{n}\right) \times Q$. Then there exists a homeomorphism $\hat{h}: E^{n} \times Q \rightarrow$ $E^{n} \times Q$ such that $\hat{h} \mid \operatorname{Bd}\left(E^{n}\right) \times Q=$ id and $\hat{h}|A=h| A$.

Proof. Let $\tilde{A}=\left(\left(e^{n} \times \mathrm{id}\right) \mid B_{1}^{n} \times Q\right)^{-1}(A)$, which is a subset of Int $\left(B_{1}^{n}\right) \times Q$. We will now show that there exists a covering transformation $\phi: R^{n} \times Q \rightarrow R^{n} \times Q$ such that $\phi \widetilde{h}(\widetilde{A}) \subset \operatorname{Int}\left(B_{1}^{n}\right) \times Q$. To see this note that

$$
\alpha=\left(\left(e^{n} \times \mathrm{id}\right) \mid B_{1}^{n} \times Q\right)^{-1} h \mid A: A \rightarrow \operatorname{Int}\left(B_{1}^{n}\right) \times Q
$$

qives a lifting of $h \mid A: A \rightarrow \operatorname{Int}\left(E^{n}\right) \times Q$. Choose any $a_{0} \in A$ and note that $\alpha\left(a_{0}\right)=\left(\left(e^{n} \times \mathrm{id}\right) \mid B_{1}^{n} \times Q\right)^{-1} h\left(a_{0}\right)=\widetilde{a}_{0} \in \operatorname{Int}\left(B_{1}^{n}\right) \times Q$. Now

$$
\beta=\widetilde{h}\left(\left(e^{n} \times \mathrm{id}\right) \mid B^{n} \times Q\right)^{-1} \mid A: A \rightarrow R^{n} \times Q
$$

also gives a lifting of $h \mid A: A \rightarrow \operatorname{Int}\left(E^{n}\right) \times Q$. Let $\phi: R^{n} \times Q \rightarrow R^{n} \times Q$ be a covering transformation which satisfies $\phi \beta\left(a_{0}\right)=\widetilde{a}_{0}$. Then we must have $\phi \beta=\alpha$, since $A$ is connected. Thus $\phi \widetilde{h}(\widetilde{A}) \subset \operatorname{Int}\left(B_{1}^{n}\right) \times Q$. We also note that $\phi \tilde{h}$ is bounded. So without loss of generality we man assume that $\widetilde{h}$ satisfies $\widetilde{h}(\widetilde{A}) \subset \operatorname{Int}\left(B_{1}^{n}\right) \times Q$. We will now construct 
a homeomorphism $f: B_{1}^{n} \times Q \rightarrow B_{1}^{n} \times Q$ such that $f \mid \operatorname{Bd}\left(B_{1}^{n}\right) \times Q=$ id and $f|\widetilde{A}=\widetilde{h}| \widetilde{A}$. Once this is done we can get our required $\hat{h}$ by defining

$$
\hat{h}=\left(e^{n} \times \mathrm{id}\right) f\left(\left(e^{n} \times \mathrm{id}\right) \mid B_{1}^{n} \times Q\right)^{-1} .
$$

Since $\widetilde{A}$ is compact we can find $t, 0<t<1$, such that

$$
\widetilde{A} \cup \widetilde{h}(\widetilde{A}) \subset(-t, t)^{n} \times Q \text {. }
$$

Let $\gamma:$ Int $\left(B_{1}^{n}\right) \rightarrow R^{n}$ be a radial expansion which satisfies $\gamma \mid B_{t}^{n}=\mathrm{id}$. Let $C\left(B_{1}^{n} \times Q\right)=\left(\operatorname{Int}\left(B_{1}^{n}\right) \times Q\right) \cup\left(\operatorname{Bd}\left(B_{1}^{n}\right) \times\{0\}\right)$ and let $p: B_{1}^{n} \times Q \rightarrow$ $C\left(B_{1}^{n} \times Q\right)$ be defined by $p \mid \operatorname{Int}\left(B_{1}^{n}\right) \times Q=$ id and $p(x, q)=(x, 0)$ for all $(x, q) \in \mathrm{Bd}\left(B_{1}^{n}\right) \times Q$. Give $C\left(B_{1}^{n} \times Q\right)$ the identification topology determined by $p$. Since $Q$ is known to be homeomorphic to its own cone, it follows that $C\left(B_{1}^{n} \times Q\right) \cong Q$. Define a homeomorphism $g$ : $C\left(B_{1}^{n} \times Q\right) \rightarrow C\left(B_{1}^{n} \times Q\right)$ by

$$
g= \begin{cases}\mathrm{id} & \text { on } \operatorname{Bd}\left(B_{1}^{n}\right) \times\{0\} \\ (\gamma \times \mathrm{id})^{-1} \tilde{h}(\gamma \times \mathrm{id}) & \text { on } \operatorname{Int}\left(B_{1}^{n}\right) \times Q .\end{cases}
$$

It is clear that $g$ is a homeomorphism because $\tilde{h}$ is bounded. Note that $g|\widetilde{A}=\widetilde{h}| \widetilde{A}$. Choose a homeomorphism $\delta: B_{1}^{n} \times Q \rightarrow C\left(B_{1}^{n} \times\right.$ $Q)$ which satisfies $\delta \mid B_{t}^{n} \times Q=\mathrm{id}$ and $\delta$ is close enough to $p$ so that $\delta\left(\operatorname{Bd}\left(B_{1}^{n}\right) \times Q\right) \cap\left(B_{t}^{n} \times Q\right)=\varnothing$. Then $\delta$ induces a homeomorphism $\hat{g}: B_{1}^{n} \times Q \rightarrow B_{1}^{n} \times Q$ which makes the following diagram commute.

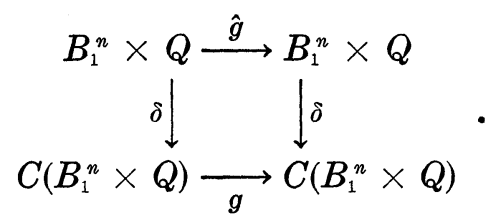

Note that $\hat{g}|\tilde{A}=g| \tilde{A}=h \mid \tilde{A}$. If $\delta$ is chosen sufficiently close to $p$ then we have $\hat{g} \mid \mathrm{Bd}\left(B_{1}^{n}\right) \times Q \cong \mathrm{id}$ (in $\left.\left(B_{1}^{n} \backslash B_{t}^{n}\right) \times Q\right)$. Using the fact that $\mathrm{Bd}\left(B_{1}^{n}\right) \times Q$ is a $Z$-set in $B_{1}^{n} \times Q$, the Homeomorphism Extension Theorem of $\S 2$ applies to give us a homeomorphism $\theta: B_{1}^{n} \times Q \rightarrow B_{1}^{n} \times$ $Q$ such that $\theta \mid B_{t}^{n} \times Q=\mathrm{id}$ and $\theta\left|\mathrm{Bd}\left(B_{1}^{n}\right) \times Q=\hat{g}\right| \mathrm{Bd}\left(B_{1}^{n}\right) \times Q$. Then $f=\theta^{-1} \hat{g}$ clearly fulfills our requirements.

7. Handle straightening. In this section we obtain our infinitedimensional version of the handle-straightening results of KirbySiebenmann. The statement of the result is given in Theorem 7.1.

Let $M$ be a finite-dimensional $P L$ manifold and let $h: B_{2}^{n} \times Q \rightarrow M \times Q$ be an embedding such that $\mathrm{Bd}\left(h\left(B_{2}^{n} \times Q\right)\right)=h\left(\operatorname{Bd}\left(B_{2}^{n}\right) \times Q\right)$. Then we 
say that $h$ can be straightened if there exists a homeomorphism $f: B_{2}^{n} \times$ $Q \rightarrow B_{2}^{n} \times Q$ and an integer $k>0$ such that $f \mid \mathrm{Bd}\left(B_{2}^{n}\right) \times Q=\mathrm{id}$ and $h f\left(B_{1 / 2}^{n} \times Q\right)=N \times Q_{k+1}$, where $N$ is a $P L$ submanifold of $M \times I^{k}$ such that $\mathrm{Bd}(N)$ is a $P L$ submanifold of $M \times I^{k}$ which is $P L$ bicollared.

THEOREM 7.1. Let $M$ be a finite-dimensional $P L$ manifold and let $h: B_{2}^{n} \times Q \rightarrow M \times Q$ be an embedding such that $\mathrm{Bd}\left(h\left(B_{2}^{n} \times Q\right)\right)=$ $h\left(\mathrm{Bd}\left(B_{2}^{n}\right) \times Q\right)$. If $n \geqq 2$, then $h$ can be straightened.

Proof. We use the notation $D^{n}$ and $E^{n}$ of $\S 5$. Using Lemma 5.2 let $\alpha:\left(T^{n} \backslash D^{n}\right) \times Q \rightarrow \operatorname{Int}\left(B_{2}^{n}\right) \times Q$ be an immersion such that $\alpha\left(e^{n} \times \mathrm{id}\right) \mid B_{1}^{n} \times Q=\mathrm{id}$. Then it follows that $\alpha \mid E^{n} \times Q$ is one-to-one. Also $h \alpha:\left(T^{n} \backslash D^{n}\right) \times Q \rightarrow M \times Q$ is an immersion. Let

$$
\begin{aligned}
& K_{0}=e^{n}\left(B_{3 / 4}^{n}\right) \times Q, \\
& G=e^{n}\left(\operatorname{Int}\left(B_{1}^{n}\right)\right) \times Q, \\
& K=\left(T^{n} \backslash e^{n}\left((1.5,3.5)^{n}\right)\right) \times Q .
\end{aligned}
$$

Note that $K_{0} \subset G \subset K$, where $K_{0}$ and $K$ are compact and $G$ is open. Moreover we see that $h \alpha \mid G$ is one-to-one. Using Lemma 5.1 there exists a $P L$ manifold $N$, a compact $P L$ submanifold $N_{0}$ of $N$, and an embedding $\phi: N \times Q \rightarrow\left(T^{n} \backslash D^{n}\right) \times Q$ such that

(1) $K_{0} \subset \operatorname{Int}\left(\phi\left(N_{0} \times Q\right)\right) \subset \dot{\phi}\left(N_{0} \times Q\right) \subset G \subset K \subset \dot{\phi}(N \times Q)$,

(2) there exists an integer $m>0$ and a $P L$ submanifold $M_{0}$ of $M \times I^{m}$ such that $h \alpha \dot{\phi}\left(N_{0} \times Q\right)=M_{0} \times Q_{m+1}$,

(3) $h \alpha \phi \mid N_{0} \times\{0\}: N_{0} \times\{0\} \rightarrow M_{0} \times\{0\}$ is a $P L$ homeomorphism,

(4) for any $\left(n,\left(q_{i}\right)\right) \in N_{0} \times Q$ we have $h \alpha^{\prime} \phi\left(n,\left(q_{i}\right)\right)=\left(m,\left(q_{i}\right)\right)$, where $m$ is chosen so that $h \alpha \phi(n, 0)=(m, 0)$.

Note that $\phi^{-1} \mid \operatorname{Int}\left(K_{0}\right): \operatorname{Int}\left(K_{0}\right) \rightarrow \operatorname{Int}\left(N_{0}\right) \times Q$ is an open embedding. Using Theorem 3.2 there exists an integer $k_{1}>0$ and a compact contractible PL submanifold $Y_{1}$ of $\operatorname{Int}\left(N_{0}\right) \times I^{k_{1}}$ such that

(1) $\mathrm{Bd}\left(Y_{1}\right)$ is a $P L$ submanifold of $\operatorname{Int}\left(N_{0}\right) \times I^{k_{1}}$ which is $P L$ bicollared,

(2) $\phi^{-1}\left(e^{n}\left(B_{1 / 2}^{n}\right) \times Q\right) \subset \operatorname{Int}\left(Y_{1}\right) \times Q_{k_{1}+1} \subset Y_{1} \times Q_{k_{1}+1}$

$$
\subset \phi^{-1}\left(e^{n}\left(\operatorname{Int}\left(B_{2 / 3}^{n}\right)\right) \times Q\right),
$$

(3) the inclusion $\left.\mathrm{Bd}\left(Y_{1}\right) \times Q_{k_{1}+1} \subset \phi^{-1}\left(e^{n}\left(B_{2 / 3}^{n}\right) \operatorname{Int}\left(B_{1 / 2}^{n}\right)\right) \times Q\right)$ is a homotopy equivalence.

It follows from the construction of $\phi$ that $h \alpha \dot{\phi}\left(Y_{1} \times\{0\}\right)$ is a $P L$ subspace of $M \times I^{m+k_{1}} \times\{0\} \subset M \times Q$ (where $Y_{1} \times\{0\} \subset\left(N_{0} \times I^{k_{1}}\right) \times$ $\left.Q_{k_{1}+1}\right)$. We will construct a homeomorphism $f: B_{2}^{n} \times Q \rightarrow B_{2}^{n} \times Q$ such that $f \mid \mathrm{Bd}\left(B_{2}^{n}\right) \times Q=i d$ and $f\left(B_{1 / 2}^{n} \times Q\right)=\alpha \dot{\phi}\left(Y_{1} \times Q_{k_{1}+1}\right)$. This will fulfill our requirements. In order to construct $f$ we will first construct a homeomorphism $\tilde{f}: E^{n} \times Q \rightarrow E^{n} \times Q$ such that $\tilde{f} \mid \mathrm{Bd}\left(E^{n}\right) \times Q=$ $i d$ and $\widetilde{f}\left(e^{n}\left(B_{1 / 2}^{n}\right) \times Q\right)=\dot{\phi}\left(Y_{1} \times Q_{k_{1}+1}\right)$. Once we have $\tilde{f}$ we can define 
$f$ by

$$
f= \begin{cases}\operatorname{id} & \text { on }\left(B_{2}^{n} \backslash B_{1}^{n}\right) \times Q \\ \alpha \widetilde{f}\left(\alpha \mid E^{n}\right)^{-1} & \text { on } B_{1}^{n} \times Q\end{cases}
$$

It is clear that $\phi^{-1} \mid e^{n}\left((1.3,3.7)^{n} \backslash[1.4,3.6]^{n}\right) \times Q$ gives an open embedding of $e^{n}\left((1.3,3.7)^{n} \backslash[1.4,3.6]^{n}\right) \times Q$ into $N \times Q$. Using Theorem 3.1 we can find an integer $k_{2} \geqq k_{1}$ and a compact $P L$ submanifold $Y_{2}$ of $N \times I^{k_{2}}$ such that

(1) $Y_{2}$ is $P L$ bicollared,

(2) $Y_{2} \times Q_{k_{2}+1} \subset \phi^{-1}\left(e^{n}\left((1.3,3.7)^{n} \backslash[1.4,3.6]^{n}\right) \times Q\right)$,

(3) the above inclusion is a homotopy equivalence,

(4) $Y_{2} \times Q_{k_{2}+1}$ separates $\dot{\phi}^{-1}\left(e^{n}\left([1.3,3.7]^{n} \backslash(1.4,3.6)^{n}\right) \times Q\right)$ into two components, one containing $\dot{\phi}^{-1}\left(e^{n}\left(\mathrm{Bd}\left([1.3,3.7]^{n}\right)\right) \times Q\right)$ and the other containing $\phi^{-1}\left(e^{n}\left(\mathrm{Bd}\left([1.4,3.6]^{n}\right)\right) \times Q\right)$.

It is easily seen that $\varphi\left(Y_{2} \times Q_{k_{2}+1}\right)$ separates $T^{n} \times Q$ into two components $A$ and $B$, where $\mathrm{Cl}(A)$ (the closure of $A$ ) contains $e^{n}([1.4$, $\left.3.6]^{n}\right) \times Q$ and $\mathrm{Cl}(B)$ contains $\left(T^{n} \times Q\right) \backslash\left(e^{n}\left((1.3,3.7)^{n}\right) \times Q\right)$. Let $A^{\prime}, B^{\prime}$ denote the closures of the intersections of $A, B$ with $e^{n}\left([1.3,3.7]^{n} \backslash\right.$ $\left.(1.4,3.6)^{n}\right) \times Q$, respectively. Then $\varphi^{-1}\left(A^{\prime}\right)$ and $\varphi^{-1}\left(B^{\prime}\right)$ are the closures of the components of (4) above. Recalling the comments concerning ANR's made at the end of the proof of Lemma 3.5, there exists a strong deformation retraction of $A^{\prime} \cup B^{\prime}$ onto $\varphi\left(Y_{2} \times Q_{k_{2}+1}\right)$; let $D_{t}: A^{\prime} \cup B^{\prime} \rightarrow$ $A^{\prime} \cup B^{\prime}, 0 \leqq t \leqq 1$, be a homotopy which gives such a strong deformation retraction, where $D_{0}=\mathrm{id}$ and $D_{1}$ is a retraction of $A^{\prime} \cup B^{\prime}$ onto $\varphi\left(Y_{2} \times Q_{k_{2}+1}\right)$. Let $r: A^{\prime} \cup B^{\prime} \rightarrow B^{\prime}$ be the retraction defined by $r \mid B^{\prime}=$ id and $r\left|A^{\prime}=D_{1}\right| A^{\prime}$. Then define a homotopy $D_{t}^{\prime}: B^{\prime} \rightarrow B^{\prime}$ by $D_{t}^{\prime}=r D_{t} \mid B^{\prime}$, for $0 \leqq t \leqq 1$. It is clear that $D_{t}^{\prime}$ defines a strong deformation retraction of $B^{\prime}$ onto $\varphi\left(Y_{2} \times Q_{k_{2}+1}\right)$. Using $D_{t}^{\prime}$ it follows that $C l(A)$ is contractible, and it is clearly a $Q$-manifold. But it is known that all compact, contractible $Q$-manifolds are homeomorphic to $Q$ [4]. Thus $C l(A) \cong Q$. [One is tempted to apply Lemma 4.1 here, but it will not work because it is not known that $\mathrm{Cl}(A)$ is triangulated.] We can easily use similar tricks to show that the inclusion

$$
\left(T^{n} \times Q\right) \backslash\left(e^{n}\left((1.3,3.7)^{n}\right) \times Q\right) \subset \mathrm{Cl}(B)
$$

is a homotopy equivalence.

Let $Y_{1}^{\prime}=Y_{1} \times I_{k_{1}+1} \times \cdots \times I_{k_{2}}$ and let $Y_{2}^{\prime}=p_{k_{2}} \phi^{-1}(\mathrm{Cl}(A))$, where $p_{k_{2}}: N \times Q \rightarrow N \times I^{k_{2}}$ is the projection mapping. Put $R=\left(N \times I^{k_{2}}\right) \backslash$ (Int $\left(Y_{1}^{\prime}\right) \cup \operatorname{Int}\left(Y_{2}^{\prime}\right)$ ). Then $R$ is a compact $P L$ submanifold of $N \times I^{k_{2}}$ and $\mathrm{Bd}(R)=\mathrm{Bd}\left(Y_{2}^{\prime}\right) \cup \mathrm{Bd}\left(Y_{2}^{\prime}\right)$. It follows (as above) that if 


$$
C=\left[T^{n} \backslash e^{n}\left((1.3,3.7)^{n} \cup \operatorname{Int}\left(B_{2 / 3}^{n}\right)\right)\right] \times\{0\} \subset \phi\left(R \times Q_{k_{2}+1}\right),
$$

then the inclusion $i: C \subset \phi\left(R \times Q_{k_{2}+1}\right)$ is a homotopy equivalence. Let

$$
j: C \subset\left[T^{n} \backslash\left(\operatorname{Int}\left(D^{n}\right) \cup e^{n}\left(\operatorname{Int}\left(B_{1 / 2}^{n}\right)\right)\right)\right] \times Q
$$

denote inclusion, which is also a homotopy equivalence.

If $n \geqq 3$, then $\pi_{1}(C)=Z \oplus Z \oplus \cdots \oplus Z(n$ copies of $Z)$. If $n=$ 2 , then it easily follows that $\pi_{1}(C)=Z^{*} Z^{*} Z$ (where ${ }^{*}$ denotes free product). In any case we have $\mathrm{Wh}\left(\pi_{1}(C)\right)=0$ (see [13], page 373, for references). Using Theorem 4.1 we can find a homeomorphism

$$
\gamma^{\prime}:\left[T^{n} \backslash\left(\operatorname{Int}\left(D^{n}\right) \cup e^{n}\left(\operatorname{Int}\left(B_{1 / 2}^{n}\right)\right)\right)\right] \times Q \rightarrow \phi\left(R \times Q_{k_{2}+1}\right)
$$

such that $\gamma^{\prime} \mid C=\mathrm{id}$. Using the Homeomorphism Extension Theorem of $\S 2$ we can adjust $\gamma^{\prime}$ to get a homeomorphism

$$
\gamma:\left[T^{n} \backslash\left(\operatorname{Int}\left(D^{n}\right) \cup e^{n}\left(\operatorname{Int}\left(B_{1 / 2}^{n}\right)\right)\right)\right] \times Q \rightarrow \phi\left(R \times Q_{k_{2}+1}\right)
$$

which satisfies

$$
\gamma \mid C=\mathrm{id}, \gamma\left(\operatorname{Bd}\left(D^{n}\right) \times Q\right)=\phi\left(Y_{2} \times Q_{k_{2}+1}\right),
$$

and

$$
\gamma\left(\operatorname{Bd}\left(e^{n}\left(B_{1 / 2}^{n}\right)\right) \times Q\right)=\dot{\phi}\left(\operatorname{Bd}\left(Y_{1}^{\prime}\right) \times Q_{k_{2}+1}\right) .
$$

Note that $T^{n} \times Q=\phi\left(R \times Q_{k_{2}+1}\right) \cup \mathrm{Cl}(A) \cup \dot{\phi}\left(Y_{1}^{\prime} \times Q_{k_{2}+1}\right)$, where $\mathrm{Cl}(A)$ has already been observed to be a Hilbert cube and $\varphi\left(Y_{1}^{\prime} \times Q_{k_{2}+1}\right)$ is a Hilbert cube by Lemma 4.1. By applying the Homeomorphism Extension Theorem again to

$$
\gamma \mid \mathrm{Bd}\left(D^{n}\right) \times Q: \operatorname{Bd}\left(D^{n}\right) \times Q \rightarrow \mathrm{Bd}(\mathrm{Cl}(A))
$$

and

$$
\gamma \mid \mathrm{Bd}\left(e^{n}\left(B_{1 / 2}^{n}\right)\right) \times Q: \mathrm{Bd}\left(e^{n}\left(B_{1 / 2}^{n}\right)\right) \times Q \rightarrow \phi\left(\mathrm{Bd}\left(Y_{1}^{\prime}\right) \times Q_{k_{2}+1}\right),
$$

it easily follows that $\gamma$ can be extended to a homeomorphism

$$
\delta: T^{n} \times Q \rightarrow T^{n} \times Q .
$$

Using Lemma 6.2 it follows that $\delta$ can be covered by a bounded homeomorphism $\tilde{\delta}: R^{n} \times Q \rightarrow R^{n} \times Q$. Using Theorem 6.1 the existence of our required $\tilde{f}$ now follows.

8. Proof of the theorem. We are given a triangulated $Q$-manifold $X$ and an open embedding $h: R^{n} \times Q \rightarrow X$, where $n \geqq 2$. We want to prove that $X \backslash h\left(\operatorname{Int}\left(B_{1}^{n}\right) \times Q\right)$ is a triangulated $Q$-manifold.

Let $\phi: X \rightarrow|K| \times Q$ be a homeomorphism, for some countable 
locally-finite simplicial complex $K$. Then there exists a finite subcomplex $K_{0}$ of $K$ such that $\phi h\left(B_{2}^{n} \times Q\right) \subset \operatorname{Int}\left(\left|K_{0}\right| \times Q\right)$ and $\operatorname{Bd}\left(\left|K_{0}\right|\right)$ is a $P L$ subspace of $|K|$ which is $P L$ bicollared. Let us regard $\left|K_{0}\right|$ as a $P L$ subspace of some $I^{n}$ (where $\left.I^{n} \cap|K|=\left|K_{0}\right|\right)$, with $\mathrm{Bd}\left(\left|K_{0}\right|\right) \subset$ $\mathrm{Bd}\left(I^{n}\right)$ and $\operatorname{Int}\left(\left|K_{0}\right|\right) \subset \operatorname{Int}\left(I^{n}\right)$. Choose a regular neighborhood $M$ of $\left|K_{0}\right|$ in $I^{n}$ which meets $\mathrm{Bd}\left(I^{n}\right)$ regularly. Then we get a new complex $|L|=M \cup\left(|K||| K_{0} \mid\right)$, which is a countable locally-finite simplicial complex containing $M$ as a $P L$ subspace.

It follows from [17] that there exists a homeomorphism $\psi:|L| \times$ $Q \rightarrow|K| \times Q$ such that $\psi$ is the identity on $(|L| \mid M) \times Q$. Then $\psi^{-1} \dot{\phi}: X \rightarrow|L| \times Q$ is a homeomorphism which satisfies

$$
\psi^{-1} \phi h\left(B_{2}^{n} \times Q\right) \subset \operatorname{Int}(M) \times Q .
$$

Since $M$ is a $P L$ manifold our result now follows from Theorem 7.1.

\section{REFERENCES}

1. R. D. Anderson, On topological infinite deficiency, Michigan Math. J., 14 (1967), 365-383.

2. R. D. Anderson and T. A. Chapman, Extending homeomorphisms to Hilbert cube manifolds, Pacific J. of Math., 38 (1971), 281-293.

3. W. Browder, Structures on $M \times R$, Proc. Cam. Phil. Soc., 61 (1965), 337-345.

4. T. A. Chapman, On the structure of Hilbert cube manifolds, Composition Math., 24 (1972), 329-353.

5. - Compact Hilbert cube manifolds and the invariance of Whitehead torsion, Bull. Amer. Math. Soc., to appear.

6. Topological invariance of Whitehead torsion, Amer. J. Math., to appear.

7. M. Cohen, Simplical structures and transverse cellularity, Annals of Math., 85 (1967), 218-245.

8. J. F. P. Hudson, Piecewise Linear Topology, W. A. Benjamin, Inc., New York, 1969.

9. R. C. Kirby, Lectures on Triangulation of Manifolds, Mimeo. University of California, Los Angeles, 1969.

10. R. C. Kirby and L. C. Siebenmann, On the triangulation of manifolds and the hauptvermutung, Bull. Amer. Math. Soc., 75 (1969), 742-749.

11. A. G. Kurosh, The Theory of Groups, Volume two, Chelsea Publishing Company, New York, 1960.

12. W. S. Massey, Algebraic Topology, Harcourt, Brace and World, Inc., New York, 1967.

13. J. Milnor, Whitehead torsion, Bull. Amer. Math. Soc., 72 (1966), 358-426.

14. L. C. Siebenmann, The obstruction to finding a boundary for an open manifold of dimension greater than five, Thesis, Princeton University, Princeton, N. J., 1965.

15. E. H. Spanier, Algebraic Topology, McGraw-Hill, Inc., New York, 1966.

16. C. T. C. Wall, Finiteness conditions for $C W$-complexes, Annals of Math., 81 (1965), $56-69$.

17. J. E. West, Infinite products which are Hilbert cubes, Trans. Amer. Math. Soc., 150 (1970), 1-25.

18. J. H. C. Whitehead, Simple homotopy types, Amer. J. Math., 72 (1950), 1-57.

Received June 12, 1972. Supported in part by NSF Grant GP-28374. 



\section{PACIFIC JOURNAL OF MATHEMATICS}

EDITORS

\author{
H. SAMELSON \\ Stanford University \\ Stanford, California 94305 \\ C. R. HOBBY \\ University of Washington \\ Seattle, Washington 98105
}

\author{
J. DUGUNDJI \\ Department of Mathematics \\ University of Southern California \\ Los Angeles, California 90007 \\ RICHARD ARENS \\ University of California \\ Los Angeles, California 90024
}

\section{ASSOCIATE EDITORS}

E. F. BECKENBACH

B. H. NeUManN

F. WOLF

K. YosHIDA

\section{SUPPORTING INSTITUTIONS}

UNIVERSITY OF BRITISH COLUMBIA

CALIFORNIA INSTITUTE OF TECHNOLOGY

UNIVERSITY OF CALIFORNIA

MONTANA STATE UNIVERSITY

UNIVERSITY OF NEVADA

NEW MEXICO STATE UNIVERSITY

OREGON STATE UNIVERSITY

UNIVERSITY OF OREGON

OSAKA UNIVERSITY
UNIVERSITY OF SOUTHERN CALIFORNIA

STANFORD UNIVERSITY

UNIVERSITY OF TOKYO

UNIVERSITY OF UTAH

WASHINGTON STATE UNIVERSITY UNIVERSITY OF WASHINGTON

AMERICAN MATHEMATICAL SOCIETY NAVAL WEAPONS CENTER 


\section{Pacific Journal of Mathematics}

\section{Vol. 45, No. $1 \quad$ September, 1973}

William George Bade, Complementation problems for the Baire classes .......... 1

Ian Douglas Brown, Representation of finitely generated nilpotent groups ........ 13

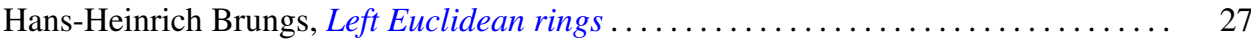

Victor P. Camillo and John Cozzens, A theorem on Noetherian hereditary rings ..... 35

James Cecil Cantrell, Codimension one embeddings of manifolds with locally flat

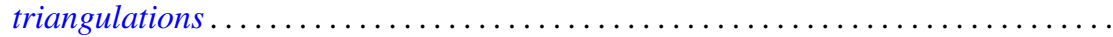

L. Carlitz, Enumeration of up-down permutations by number of rises . . . . . . . . . .

Thomas Ashland Chapman, Surgery and handle straightening in Hilbert cube

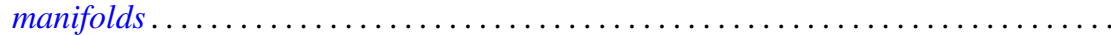

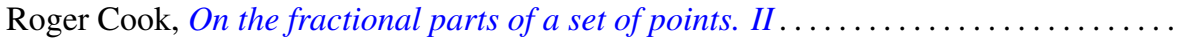

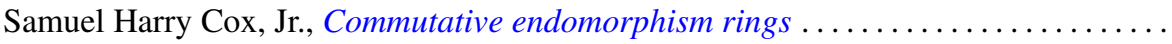

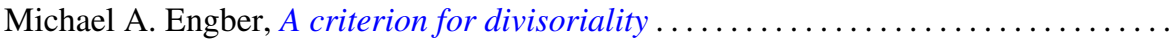

Carl Clifton Faith, When are proper cyclics injective . . . . . . . . . . . . . . 97

David Finkel, Local control and factorization of the focal subgroup . . . . . . . . . 113

Theodore William Gamelin and John Brady Garnett, Bounded approximation by

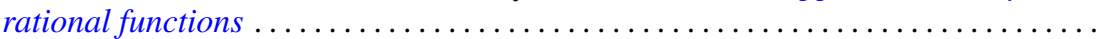

Kazimierz Goebel, On the minimal displacement of points under Lipschitzian

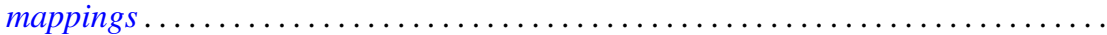

Frederick Paul Greenleaf and Martin Allen Moskowitz, Cyclic vectors for representations associated with positive definite measures: nonseparable

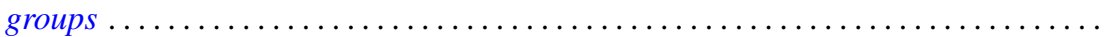

Thomas Guy Hallam and Nelson Onuchic, Asymptotic relations between perturbed linear systems of ordinary differential equations .

David Kent Harrison and Hoyt D. Warner, Infinite primes of fields and completions. .

James Michael Hornell, Divisorial complete intersections . ......

Jan W. Jaworowski, Equivariant extensions of maps ..............

John Jobe, Dendrites, dimension, and the inverse arc function .. .

Gerald William Johnson and David Lee Skoug, Feynman integrals of non-factorable

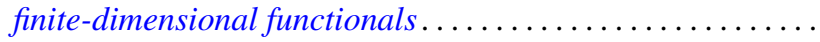

Dong S. Kim, A boundary for the algebras of bounded holomorphic functions ...... 269

Abel Klein, Renormalized products of the generalized free field and its derivatives ... 275

Joseph Michael Lambert, Simultaneous approximation and interpolation in $L_{1}$ and

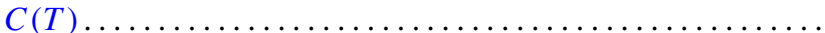

Kelly Denis McKennon, Multipliers of type $(p, p)$ and multipliers of the group

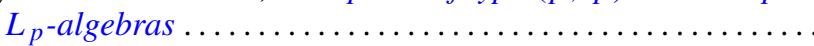

William Charles Nemitz and Thomas Paul Whaley, Varieties of implicative

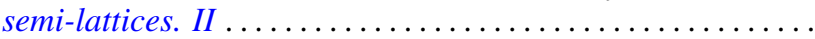

Donald Steven Passman, Some isolated subsets of infinite solvable

Norma Mary Piacun and Li Pi Su, Wallman compactifications on E-completely

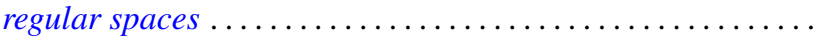

Jack Ray Porter and Charles I. Votaw, $S(\alpha)$ spaces and regular Hausdorff extensions....

Gary Sampson, Two-sided $L_{p}$ estimates of convolution transforms .

Ralph Edwin Showalter, Equations with operators forming a rig
Raymond Earl Smithson, Fixed points in partially ordered sets .

Victor Snaith and John James Ucci, Three remarks on symmetric products and

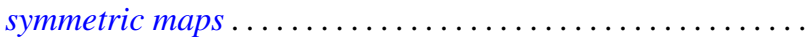

